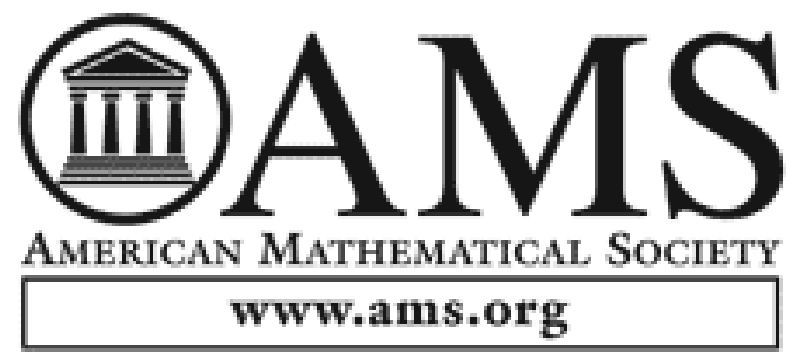

The Structure of $\sigma$-Ideals of Compact Sets

Author(s): A. S. Kechris, A. Louveau and W. H. Woodin

Source: Transactions of the American Mathematical Society, Vol. 301, No. 1 (May, 1987), pp. 263-288

Published by: American Mathematical Society

Stable URL: http://www.jstor.org/stable/2000338

Accessed: 20/05/2013 13:09

Your use of the JSTOR archive indicates your acceptance of the Terms \& Conditions of Use, available at http://www.jstor.org/page/info/about/policies/terms.jsp

JSTOR is a not-for-profit service that helps scholars, researchers, and students discover, use, and build upon a wide range of content in a trusted digital archive. We use information technology and tools to increase productivity and facilitate new forms of scholarship. For more information about JSTOR, please contact support@jstor.org. 


\title{
THE STRUCTURE OF $\sigma$-IDEALS OF COMPACT SETS
}

\author{
A. S. KECHRIS ${ }^{1}$, A. LOUVEAU AND W. H. WOODIN ${ }^{1}$
}

\begin{abstract}
Motivated by problems in certain areas of analysis, like measure theory and harmonic analysis, where $\sigma$-ideals of compact sets are encountered very often as notions of small or exceptional sets, we undertake in this paper a descriptive set theoretic study of $\sigma$-ideals of compact sets in compact metrizable spaces. In the first part we study the complexity of such ideals, showing that the structural condition of being a $\sigma$-ideal imposes severe definability restrictions. A typical instance is the dichotomy theorem, which states that $\sigma$-ideals which are analytic or coanalytic must be actually either complete coanalytic or else $G_{\delta}$. In the second part we discuss (generators or as we call them here) bases for $\sigma$-ideals and in particular the problem of existence of Borel bases for coanalytic non-Borel $\sigma$-ideals. We derive here a criterion for the nonexistence of such bases which has several applications. Finally in the third part we develop the connections of the definability properties of $\sigma$-ideals with other structural properties, like the countable chain condition, etc.
\end{abstract}

In this paper we study the descriptive set theoretic properties of $\sigma$-ideals of compact sets (in compact metrizable spaces). Such $\sigma$-ideals occur very frequently in various parts of analysis, as "smallness" notions or "exceptional" sets. Usually a lot of information about these notions comes from the structural properties inherent in the special context in which these $\sigma$-ideals are studied, but it turns out that the purely descriptive set theoretic approach is enough to give nontrivial information about these objects.

The starting point of our investigations was a recent result of Solovay $[\mathbf{S}]$ and independently Kaufman $[\mathbf{K 1}]$ about the $\sigma$-ideal of compact sets of uniqueness, which is shown to be a complete $\boldsymbol{\Pi}_{1}^{1}$ (=coanalytic) set. A set of uniqueness is a subset of the unit circle $\mathbf{T}$ for which every trigonometric series $\sum c_{n} e^{i n x}$ converging to 0 outside the set is identically 0 . (Other examples of $\sigma$-ideals of this kind that were known earlier are: the compact subsets of $\mathbf{Q}$, the countable compact subsets of $\mathbf{R}$, etc.) Heuristically this kind of result rules out in general potential criteria for characterizing when a compact set is in the $\sigma$-ideal if of a too simple form-here Borel, which is usually the proposed form.

In the first part of this paper we study systematically the possible complexity of $\sigma$-ideals of compact sets. As it happens there are essentially only two possibilities, within the analytic or coanalytic ones: Apart from trivial cases they must be either true $G_{\delta}$ sets or else true coanalytic sets. These results extend an older result of Christensen [Chr] and Saint-Raymond [StR1] on $\infty$-ideals, i.e. sets of the form $\mathcal{K}(A)$ for some subset $A$ of a compact metrizable space. (Here $\mathcal{K}(A)$ is the set of compact subsets of $A$.) They proved that if $\mathcal{K}(A)$ is analytic then $A$ (and hence

Received by the editors October 15, 1985.

1980 Mathematics Subject Classification (1985 Revision). Primary 03E15, 28A05, 28A12, 42 A63.

${ }^{1}$ Partially supported by NSF Grant DMS-8416349. 
$\mathcal{K}(A))$ is a $G_{\delta}$ set. Our results also extend for $\infty$-ideals throughout the projective hierarchy (using strong axioms of set theory), showing that the only possible classes for $\mathcal{K}(A), A$ projective, are the $\Pi_{n}^{1}$.

Once one knows that a $\sigma$-ideal is too complicated to admit simple criteria, one can search for simple criteria for generating the $\sigma$-ideal. This is the "basis problem" that we discuss in the second part. (A basis for a $\sigma$-ideal is a subset of the ideal which generates it as a $\sigma$-ideal.) Again we show that the existence of an analytic basis for a $\sigma$-ideal of compact sets implies the existence of a $G_{\delta}$ basis. Of particular interest is the problem of the existence of Borel bases for true $\Pi_{1}^{1} \sigma$-ideals. This turns out to be equivalent to a classification problem, namely whether the class of compact sets which are locally in the $\sigma$-ideal is Borel or not. We prove a sufficient criterion for nonexistence of Borel bases of true $\Pi_{1}^{1} \sigma$-ideals, which has several applications. It can be used for example to classify completely the $\Pi_{1}^{1} \infty$-ideals which have a Borel basis: An $\infty$-ideal $\mathcal{K}(A), A$ coanalytic, has a Borel basis iff $A$ is the difference of two $G_{\delta}$ sets. It implies also for instance that any "sufficiently nice" true $\Pi_{1}^{1} \sigma$-ideal which contains the 0 sets of a "continuous" capacity cannot have a Borel basis. This in turn can be used to provide interesting examples of $\Pi_{1}^{1} \sigma$-ideals with no Borel bases. The problem of developing further methods for demonstrating the nonexistence of Borel bases for $\Pi_{1}^{1} \sigma$-ideals is extremely interesting, especially in view of the important unsolved problem of the existence of a Borel basis for the $\sigma$-ideal of the compact sets of uniqueness.

In the third part of the paper, we relate the descriptive set theoretic properties of $\sigma$-ideals to other structural properties. One of them is the notion of thinness of $\sigma$-ideals: It corresponds (dually) to the countable chain condition. In potential theory, or more generally when capacities are involved, these notions have been extensively studied (see Dellacherie [D1]), and the link between thinness, descriptive set theoretical properties, and approximation properties was noticed by some authors, mainly Dellacherie and Feyel, see [DFM and DM]. We give here a general "abstract" treatment of thinness, generalizing the results known in the case of capacities, and showing that these results have very few relations with the particular properties of measures and capacities. We also introduce a descriptive set theoretic analog of "control", generalizing the concept of a set of measures being controlled by a measure. (A set of measures $S$ is controlled by a measure $\mu$ if $\forall \nu \in S(\nu \ll \mu)$.) We show for instance that controlled $\Pi_{1}^{1} \sigma$-ideals are thin (i.e. satisfy the ccc) and (extending a result of Dellacherie [D3]) that they are also $G_{\delta}$. This last result implies for instance that no "sufficiently nice" true $\Pi_{1}^{1} \sigma$-ideal of compact sets can contain the zero sets of a measure. This property is for instance true for the $\sigma$-ideal of sets of uniqueness. This is a well-known theorem in the theory of these sets, but the above result reveals an underlying descriptive set theoretic "phenomenon".

Added in proof (January 1987). The methods and results of this paper have recently found several applications in the study of the $\sigma$-ideals of closed sets of uniquenss $(U)$ and extended uniqueness $\left(U_{0}\right)$; see A. S. Kechris and A. Louveau, Descriptive set theory and the structure of sets of uniqueness, forthcoming monograph, and G. Debs and J. Saint Raymond, Ensembles d'unicité et d'unicité au sens large (to appear). In particular, in relation to questions raised in our paper, it has been shown that $U$ is calibrated (Kechris-Louveau, Debs-Saint Raymond), and that $U$ has no Borel basis (Debs-Saint Raymond), while $U_{0}$ has a Borel basis (Kechris-Louveau). 


\section{Complexity of $\sigma$-ideals of compact sets.}

1.1 Preliminaries on $\sigma$-ideals. For the rest of this paper, $E$ is a compact metrizable space, and $K(E)$ the space of compact subsets of $E$ with the Hausdorff topology, in which the basic open nbhds have the form

$$
\left\{K \in \mathcal{K}(E): K \subseteq U_{0} \& K \cap U_{1} \neq \varnothing \& \cdots \& K \cap U_{n} \neq \varnothing\right\}
$$

where $U_{0}, U_{1}, \ldots, U_{n}$ are open sets in $E$. Again $\mathcal{K}(E)$ is compact metrizable with the metric

$$
\begin{aligned}
\rho(K, L) & =\sup \{d(x, K), d(y, L): x \in L \& y \in K\}, \text { if } L, K \neq \varnothing, \\
& =\operatorname{diameter}(E), \text { otherwise, }
\end{aligned}
$$

where $d$ is a metric on $E$. We will use freely various simple facts about this topology (see $[\mathbf{K u}])$ like for example:

(i) If $\bigcup: \mathcal{K}(\mathcal{K}(E)) \rightarrow \mathcal{K}(E)$ is the union function $\bigcup(L) \equiv \bigcup L \equiv \bigcup\{K: K \in$ $L\}$, then $\bigcup$ is continuous. Also the function $\bigcup: \mathcal{K}(E) \times \mathcal{K}(E) \rightarrow \mathcal{K}(E)$ given by $\bigcup(K, F)=K \cup F$ is continuous.

(ii) If $\varphi: E \rightarrow E^{\prime}$ is continous, then $\varphi^{\prime \prime}: \mathcal{K}(E) \rightarrow \mathcal{K}\left(E^{\prime}\right)$ given by $\varphi^{\prime \prime}(K) \equiv$ $\{\varphi(x): x \in K\}$ is continuous.

(iii) If $L$ is clopen in $E$ the map $\varphi: \mathcal{K}(E) \rightarrow \mathcal{K}(E)$ given by $\varphi(K)=K \cap L$ is continuous.

We will sometimes restrict our attention to 0-dimensional (0-dim) spaces $E$, i.e. totally disconnected ones. Every such space can be always considered a subspace of the Cantor set, and moreover $\mathcal{K}(E)$ is also 0 -dim (in fact $\mathcal{K}\left(2^{\omega}\right)-\{\varnothing\} \cong 2^{\omega}$ ).

If $I \subseteq \mathcal{K}(E)$ we say that $I$ is hereditary (resp. an ideal, $\sigma$-ideal, $\infty$-ideal) if $I$ is closed under $\subseteq$ (and resp. finite unions, countable unions (which are compact), arbitrary unions (which are compact)). Similar terminology will be used for other families of sets, e.g. $\sigma$-ideals of $G_{\delta}$ sets, Borel sets, etc.

If $I$ is hereditary and $A_{I}=\{x \in E:\{x\} \in I\}$, then $I \subseteq \mathcal{K}\left(A_{I}\right)$, where for $A \subseteq E$, $\mathcal{K}(A)=\{K \in \mathcal{K}(E): K \subseteq A\}$, and if $I$ is an $\infty$-ideal then $I=\mathcal{K}\left(A_{I}\right)$.

1.2 The $\forall$-propagation lemma. Let $\Gamma$ be a class of sets in compact metrizable spaces. Denote by $\Gamma(E)$ the class $\Gamma(E) \equiv \Gamma \cap \mathcal{P}(E)$. Typical examples will be the classes $\boldsymbol{\Sigma}_{1}^{0}$ (三 open), $\boldsymbol{\Pi}_{1}^{0}$ (三 compact), $\boldsymbol{\Sigma}_{2}^{0}\left(\equiv K_{\sigma}\right), \boldsymbol{\Pi}_{2}^{0}\left(\equiv G_{\delta}\right), \ldots$, Borel, $\boldsymbol{\Sigma}_{1}^{1}(\equiv$

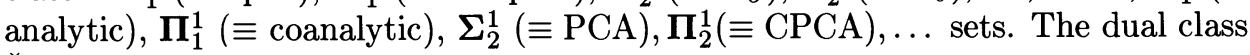
$\check{\Gamma}$ is defined by

$$
\check{\Gamma}(E)=\{E-A: A \in \Gamma(E)\} .
$$

For the rest of 1.2 we will restrict ourselves to 0 -dim compact metrizable spaces. If $\Gamma$ is a class of sets in such spaces we let $\forall \Gamma$ be the class defined by

$$
\forall \Gamma(E)=\left\{A \subseteq E: \exists B \in \Gamma\left(E \times 2^{\omega}\right) \forall x\left[x \in A \Leftrightarrow \forall y \in 2^{\omega}(x, y) \in B\right]\right\} .
$$

(Note that this notion differs from the one frequently encountered in descriptive set theory, where one works with 0-dim Polish spaces, the basic space is the Baire space $\omega^{\omega}$ and the $\forall$ operation is defined over this space.)

We call $\Gamma$ a $W a d g e$ class if for some $A \subseteq 2^{\omega}$ and for any 0-dim $E$,

$$
\Gamma(E)=\left\{B \subseteq E: \exists \text { continuous } \varphi: E \rightarrow 2^{\omega}\left(B=\varphi^{-1}[A]\right)\right\} .
$$


If $\Gamma$ is a Wadge class, and $A \subseteq E_{0}$ a 0 -dim space, we say that $A$ is $\Gamma$-hard if for every $E$ and $B \in \Gamma(E)$ there is a continuous $\varphi: E \rightarrow E_{0}$ with $B=\varphi^{-1}[A]$. If moreover $A \in \Gamma$, we call $A \Gamma$-complete. (Viewing $E_{0}$ as a subset of $2^{\omega}$, this just means that $A$ generates $\Gamma$ as a Wadge class.) Finally, we say that $A$ is a true $\Gamma$-set if $A \in \Gamma-\check{\Gamma}$.

Note that if $\Gamma$ is not self-dual, i.e. $\Gamma \neq \check{\Gamma}$ and $A$ is $\Gamma$-complete then $A$ is a true $\Gamma$-set. And for $\Gamma \subseteq$ Borel (resp. any $\Gamma$ ), Martin's Borel determinacy theorem (resp. AD) implies the converse (see e.g. $[\mathbf{M}-\mathbf{K}]$ ).

We now have

LEMMA 1 (The $\forall$-PROPAGATION LEMMA). Let $\Gamma$ be a Wadge class in 0$\operatorname{dim}$ compact metrizable spaces. If $E_{0}$ is 0 -dim compact metrizable and $A \subseteq E_{0}$ is $\Gamma$-hard, then $\mathcal{K}(A) \subseteq \mathcal{K}\left(E_{0}\right)$ is $\forall \Gamma$-hard.

Proof. Let $B \subseteq E$ be a $\forall \Gamma$ set, say $B=\forall B^{\prime}$ with $B^{\prime} \in \Gamma\left(E \times 2^{\omega}\right)$. Let $\varphi$ be continuous, $\varphi: E \times 2^{\omega} \rightarrow E_{0}$ be such that $\varphi^{-1}[A]=B^{\prime}$ and define $\psi: E \rightarrow \mathcal{K}\left(E_{0}\right)$ by $\psi(x)=\varphi^{\prime \prime}\left(\{x\} \times 2^{\omega}\right)$. Then $\psi$ is continuous and $B=\psi^{-1}[\mathcal{K}(A)]$.

In order to apply this lemma we need to know what are the Wadge classes of the form $\forall \Gamma$. The simplest Wadge classes are $\{\varnothing\},\{\varnothing\}, \boldsymbol{\Delta}_{1}^{0}\left(\equiv \boldsymbol{\Sigma}_{1}^{0} \cap \boldsymbol{\Pi}_{1}^{0} \equiv\right.$ clopen), $\Sigma_{1}^{0}, \Pi_{1}^{0}$, which are all closed under $\forall$, hence of this form. Let $D_{2}$ be (in compact metrizable spaces) the class of differences of two $\Pi_{1}^{0}$ sets, or equivalently the class of intersections of a compact and an open set. These can be also characterized as the sets which are open in their closure, and also as the locally compact metrizable spaces (see $[\mathbf{K u}]$ ). The dual class $\check{D}_{2}$ consists of unions of a compact and an open set, and the ambiguous part of this class is denoted by

$$
\Delta\left(D_{2}\right) \equiv \Delta\left(\check{D}_{2}\right) \equiv D_{2} \cap \check{D}_{2} .
$$

It is the smallest Wadge class containing $\Sigma_{1}^{0}$ and $\Pi_{1}^{0}$.

Proposition 2. We have

(i) $\forall\left(\Delta\left(D_{2}\right)\right)=D_{2}\left(=\forall D_{2}\right)$,

(ii) $\forall \check{D}_{2}=\boldsymbol{\Pi}_{2}^{0}\left(=\forall \boldsymbol{\Pi}_{2}^{0}\right)$,

(iii) $\forall \boldsymbol{\Sigma}_{2}^{0}=\boldsymbol{\Pi}_{1}^{1}\left(=\forall \boldsymbol{\Pi}_{1}^{1}\right)$,

(iv) For $n \geq 1, \forall \boldsymbol{\Sigma}_{n}^{1}=\boldsymbol{\Pi}_{n+1}^{1}\left(=\forall \boldsymbol{\Pi}_{n+1}^{1}\right)$.

Proof. For (i) notice that if $A=A_{0} \cup A_{1}$ is in $\check{D}_{2}(E), A_{0} \in \Pi_{1}^{0}, A_{1} \in \Sigma_{1}^{0}$, then $B=\left(A_{0} \times\{0\}\right) \cup\left(A_{1} \times\{1\}\right)$ is in $\Delta\left(D_{2}\right)$ in $E \times 2$ and $A=\exists B$.

For (ii) notice that if $A=\bigcup_{n} K_{n}$ is in $\Sigma_{2}^{0}(E), K_{n} \in \Pi_{1}^{0}$ then $B=\bigcup_{n}\left(K_{n} \times\{n\}\right)$ is in $D_{2}(E \times(\omega+1))$ and $A=\exists B$.

The rest is trivial.

Lemma 1 together with Proposition 2(iv) solves a problem of Dellacherie about the complexity of $\mathcal{K}(A), A \in \Sigma_{1}^{1}$. More generally if $A$ is complete $\Sigma_{n}^{1}, \mathcal{K}(A)$ is complete $\Pi_{n+1}^{1}$. And using an appropriate level of Wadge determinacy this also holds for true $\Sigma_{n}^{1}$ sets. One can ask however the following: Can it be proved in ZFC that if $A$ is true $\Sigma_{1}^{1}$ then $\mathcal{K}(A)$ is true $\boldsymbol{\Pi}_{2}^{1}$ ?

Another corollary is the following.

COROllary 3. (i) Let $E_{0}=\omega \cdot 2+1, A_{0}=\omega \cup\{\omega \cdot 2\}$. Then $\mathcal{K}\left(A_{0}\right)$ is, in $\mathcal{K}\left(E_{0}\right)$, a complete $D_{2}$ set. 
(ii) Let $E_{1}=\omega^{2}+1, A_{1}=E_{1}-\{\omega \cdot(n+1): n \in \omega\}$. Then $\mathcal{K}\left(A_{1}\right)$ is, in $\mathcal{K}\left(E_{1}\right)$, a complete $\boldsymbol{\Pi}_{2}^{0}$ set.

(iii) (Hurewicz $[\mathbf{H u}]$ ). Let $E_{2}=2^{\omega}, A_{2}=\mathbf{Q}=\left\{\alpha \in 2^{\omega}: \alpha\right.$ is eventually 0$\}$. Then $\mathcal{K}(\mathbf{Q})$ is, in $\mathcal{K}\left(E_{2}\right)$, complete $\mathbf{\Pi}_{1}^{1}$.

Proof. (i) Notice that $\mathcal{K}\left(A_{0}\right)$ is a true $D_{2}$ set. This is because if $\mathcal{K}\left(A_{0}\right)=$ $F \cup U, F \in \Pi_{1}^{0}, U \in \Sigma_{1}^{0}, F \cap U=\varnothing$, then for compact $K \subseteq A_{0}$, with $\omega \cdot 2 \in K$ we must have $K \in F$. Then $\{\omega, \omega \cdot 2\}=\lim \{n, \omega \cdot 2\}$ (in $\mathcal{K}\left(E_{0}\right)$ ), so $\{\omega, \omega \cdot 2\} \in F$, a contradiction. By Wadge Borel determinancy $\mathcal{K}\left(A_{0}\right)$ is a complete $D_{2}$ set.

(ii), (iii) Notice that $A_{1}$ is a true $\check{D}_{2}$ set in $E_{1}$ and $\mathbf{Q}$ a true $\boldsymbol{\Sigma}_{2}^{0}$ set in $E_{2}$. Then use Wadge Borel determinacy, Lemma 1 and Proposition 2.

1.3 Hurewicz-type results. A Hurewicz-type result asserts that if a set (in some space) is not in a certain class $\Gamma$, it contains as a relatively closed subset a homeomorphic copy of some fixed non- $\Gamma$-set, which could be called a Hurewicz-witness. Typically, Hurewicz's Theorem $[\mathbf{H u}]$ says that any $\Pi_{1}^{1}$ set $A$ in a compact metrizable space $E$ which is not $\Pi_{2}^{0}$ contains a closed subset homeomorphic to $\mathbf{Q}$. In fact, one can also construct a homeomorphic copy $F$ of $2^{\omega}$ inside $E$ such that $F \cap A$ is (through the homeomorphism) identified with $\mathbf{Q}$. One could also say here that the pair $\left(\mathbf{Q}, 2^{\omega}\right)$ is a Hurewicz-witness for non- $\mathbf{\Pi}_{2}^{0}$-ness. We now give a (seemingly new) proof of a sharpened and extended version of Hurewicz's theorem.

THEOREM 4. Assume $\mathrm{ZF}+\mathrm{DC}$ (resp. $+\mathrm{AD})$. Let $E$ be compact metrizable, and let $A, B$ be two disjoint subsets of $E$, with $A \in \Sigma_{1}^{1}$ (resp. arbitrary). If no $\boldsymbol{\Sigma}_{2}^{0}$ set $C$ in $E$ separates $A$ from $B$ (i.e. $A \subseteq C, C \cap B=\varnothing$ ), there is a homeomorphism $\varphi: 2^{\omega} \rightarrow F \subseteq A \cup B$, such that $\varphi^{-1}[F \cap B]=\mathbf{Q}$.

In particular, taking $B=E-A$ we obtain Hurewicz's theorem. Note also that the result for $A \in \Pi_{1}^{1}$ and $B=E-A$ needs some extra hypothesis, since $\omega_{1}=\omega_{1}^{L}$ and $A=C_{1} \subseteq 2^{\omega}$ give a counterexample. For an analysis of the set theoretical hypotheses needed for these extensions and a solution to an associated problem of Saint Raymond on characterizations of Polish spaces see the forthcoming [KLSS].

ProOF. Let $E_{C}=2^{\omega}$ and $f: E_{C} \rightarrow E$ a continuous surjection. Let $A^{\prime}=$ $f^{-1}[A], B^{\prime}=f^{-1}[B]$ and consider the following Wadge-type game: I plays $\alpha \in 2^{\omega}$, II plays $\beta \in 2^{\omega}$ and II wins iff: $\left(\alpha \in \mathbf{Q} \Rightarrow \beta \in B^{\prime}\right) \&\left(\alpha \notin \mathbf{Q} \Rightarrow \beta \in A^{\prime}\right)$. If player I has a winning strategy in this game, his strategy is a continuous function $g: E_{C} \rightarrow E_{C}$ such that $C^{\prime}=g^{-1}[\mathbf{Q}]$ separates $A^{\prime}$ from $B^{\prime}$, and thus $C=f^{\prime \prime}(C)$ is a $\Sigma_{2}^{0}$ set separating $A$ from $B$, a contradiction. So, assuming AD, player II has a winning strategy, and so there is a continuous function $\varphi: E_{C} \rightarrow E_{C}$ with $\varphi^{\prime \prime}\left(E_{C}\right) \subseteq A^{\prime} \cup B^{\prime}$ and $\varphi^{-1}\left[B^{\prime}\right]=\mathbf{Q}$. Composing with $f$ we obtain continuous $\psi: E_{C} \rightarrow E$ with $\psi^{\prime \prime}\left(E_{C}\right) \subseteq A \cup B$ and $\psi^{-1}[B]=\mathbf{Q}$. Then $F=\psi^{\prime \prime}\left(E_{C}\right)$ is compact in $E$, and $\psi^{\prime \prime}(\mathbf{Q}), \psi^{\prime \prime}\left(E_{C}-\mathbf{Q}\right)$ are disjoint dense subsets of $F$, hence $F$ is perfect. Of course, $F$ might not be 0 -dim, but an immediate construction inside $F$ (a la Cantor) gives a copy $F^{\prime}$ of $2^{\omega}$ with $F^{\prime} \cap \varphi^{\prime \prime}(\mathbf{Q})$ dense in $F^{\prime}$ and we are done.

Now to avoid $\mathrm{AD}$ in case $A$ is $\boldsymbol{\Sigma}_{1}^{1}$, we argue, working in ZF + DC only, as follows: Let $P \subseteq E_{C} \times E_{C}$ be $\Pi_{2}^{0}$ and project to $A^{\prime}$ (which is now $\Sigma_{1}^{1}$ ). In $P$, consider the largest open set $U$ whose projection $\pi U$ is $\Sigma_{2}^{0}$ separable from $B^{\prime}$. Then $P_{0}=P-U \neq \varnothing$ (since $A^{\prime}$ cannot be separated from $B^{\prime}$ by a $\Sigma_{2}^{0}$ set), and $A_{0}=\pi P_{0}$ cannot be separated from $B^{\prime}$ by a $\Sigma_{2}^{0}$ set. Let $\left\{U_{n}\right\}$ be a basis for the nonempty open subsets of $P_{0}$. By maximality of $U, \overline{\pi U_{n}} \cap B^{\prime} \neq \varnothing$, so choose 
$x_{n} \in \overline{\pi U_{n}} \cap B^{\prime}$. Let $B_{0}=\left\{x_{n}: n \in \omega\right\}$ and consider the pair $P_{0}, B_{0} \times E_{C}$ in $E_{C} \times E_{C}$. By the Baire category theorem $P_{0}$ cannot be separated from $B_{0} \times E_{c}$ by a $\Sigma_{2}^{0}$ set, because if $\bigcup_{n} K_{n}$ is such a set then for some $i, n U_{i} \subseteq K_{n}$ and so $\pi U_{i} \subseteq \pi K_{n}$ and $\overline{\pi U_{1}} \subseteq \overline{\pi K_{n}}=\pi K_{n}$, since $K_{n}$ is closed. So $x_{i} \in \pi K_{n}$ and thus $K_{n} \cap\left(B_{0} \times E_{C}\right) \neq \varnothing$, a contradiction. We can play now the Wadge-type game for $P_{0}, B_{0} \times E_{C}$ as above. Since this game is a Boolean combination of $\boldsymbol{\Pi}_{2}^{0}$ sets (note that $P_{0} \in \mathbf{\Pi}_{2}^{0}, B_{0} \in \Sigma_{2}^{0}$ ) it is determined, so, as above, player II wins. Composing his strategy with the project $\pi$ we obtain the function $\varphi$ as before.

Similar, but much easier, Hurewicz-type results hold at lower levels. Recall the pairs $\left(E_{0}, A_{0}\right),\left(E_{1}, A_{1}\right)$ defined in Corollary 3.

Proposition 5. (i) If a set $A \subseteq E$, in a compact metrizable space, is neither closed nor open, there is a homeomorphism $\varphi: \omega \cdot 2+1 \rightarrow E$ with $A_{0}=\omega \cup\{\omega \cdot 2\}=$ $\varphi^{-1}[A]$.

(ii) If a set $A \subseteq E$, in a compact metrizable space, is not in $D_{2}$, there is a homeomorphism $\varphi: \omega^{2}+1 \rightarrow E$ with $A_{1}=\omega^{2}+1-\{\omega \cdot(n+1): n \in \omega\}=\varphi^{-1}[A]$.

Proof. (i) As $A$ is not closed, it contains a discrete sequence $\left\{x_{n}\right\}$ converging to $x_{\omega} \notin A$. Similarly as $E-A$ is not closed there is a discrete sequence $\left\{x_{\omega+n}\right\}$ in $E-A$ converging to $x_{\omega \cdot 2} \in A$. Put $\varphi(\alpha)=x_{\alpha}$.

(ii) Since $A$ is not in $D_{2}$, hence not locally compact, let $x_{\omega} \in A$ have no compact neighborhood, and choose discrete disjoint sequences $\left\{x_{\omega \cdot n+m}\right\}$ in the ball $B(x, 1 /(n+1))$ in $A$ converging to distinct points $x_{\omega \cdot(n+1)}$ outside $A$. Put $\varphi(\alpha)=x_{\alpha}$ again.

Finally we quote another Hurewicz-type result due to Saint Raymond [StR2].

THEOREM 6(SAINT RAYMOND [StR2]). Let $E_{3}=2^{\omega} \times 2^{\omega}$ and $A_{3}=\{(\alpha, \beta)$ $\in E_{3}: \alpha \notin \mathbf{Q}$ or $\left.\beta \in \mathbf{Q}\right\}$. Then if a Borel set $A \subseteq E, E$ compact metrizable, is not a difference of two $\Pi_{2}^{0}$ sets, there is a homeomorphism $\varphi: E_{3} \rightarrow E$ with $\varphi^{-1}[A]=A_{3}$.

1.4 Complexity of $\sigma$-ideals. We prove now the main results about the complexity of $\Pi_{1}^{1} \sigma$-ideals.

In the results that follow, if $\Gamma$ is a class of sets in compact metrizable spaces and $A \subseteq E_{0}$ is in $\Gamma\left(E_{0}\right)$, then we will call $A \Gamma$-complete if for any 0-dim $E$ and $B \in \Gamma(E)$ there is a continuous $\varphi: E \rightarrow E_{0}$ with $B=\varphi^{-1}[A]$.

THEOREM 7. (i) Let I be a $\Pi_{1}^{1} \sigma$-ideal of compact sets in a compact metrizable space. Let $B \subseteq I$, and let $B_{\sigma}$ be the class of compact sets which are countable unions of sets in $B$. (Thus $B_{\sigma} \subseteq I$.) If there exists a $\Sigma_{1}^{1}$ set $C$ with $B_{\sigma} \subseteq C \subseteq I$, then there exists a $\Pi_{2}^{0}$ set $H$ with $B_{\sigma} \subseteq H \subseteq I$.

(ii) (The Dichotomy Theorem). Every $\Pi_{1}^{1} \sigma$-ideal of compact sets is either $\mathbf{\Pi}_{1}^{1}$ complete or else it is $\mathbf{\Pi}_{2}^{0}$.

Proof. (i) If no such $\Pi_{2}^{0}$ set $H$ exists we can apply Theorem 4 to $K(E)-I$ and $B$. This gives a compact Cantor set $F \subseteq B \cup(\mathcal{K}(E)-I)$ and $F \cap B \cong \mathbf{Q}$. Consider the continuous $\varphi: \mathcal{K}(F) \rightarrow \mathcal{K}(E)$ given by $\varphi(L)=\bigcup L$. Then for $L \in \mathcal{K}(F)$

$$
L \subseteq F \cap B \Leftrightarrow \bigcup L \in B_{\sigma} \Leftrightarrow \bigcup L \in I,
$$

so $\varphi^{-1}\left[B_{\sigma}\right]=\varphi^{-1}[I]=\mathcal{K}(F \cap B) \cong \mathcal{K}(\mathbf{Q})$, which by Corollary 3 is complete $\boldsymbol{\Pi}_{1}^{1}$. Hence no $\Sigma_{1}^{1}$ set $C$ can satisfy $B_{\sigma} \subseteq C \subseteq I$, and we are done. 
(ii) Put $B=I$ in (i), and apply the preceding proof.

It remains to look at $\Pi_{2}^{0} \sigma$-ideals. The following result completes the picture.

THEOREM 8. If I is a $\mathbf{\Pi}_{2}^{0} \sigma$-ideal of compact sets in a compact metrizable space, then either $I$ is $\Pi_{2}^{0}$-complete, or else $I$ is in $D_{2}$. In the latter case $I$ is an $\infty$-ideal and hence is of the form $\mathcal{K}(A)$ for some $A$ in $D_{2}$. In particular, any $\Pi_{2}^{0} \sigma$-ideal not of the form $K(A)$ is complete $\Pi_{2}^{0}$. And finally if $I$ is in $D_{2}$ it is either $D_{2}$-complete or else is either $\Sigma_{1}^{0}$ or $\Pi_{1}^{0}$, i.e. of the form $\mathcal{K}(A)$ for $A$ open or closed.

In fact one can prove the following stronger result about ideals in general.

THEOREM 9. If $I$ is a $\Delta_{2}^{0}$ (i.e. both $\Pi_{2}^{0}$ and $\boldsymbol{\Sigma}_{2}^{0}$ ) ideal of compact sets in a compact metrizable space, then it is an $\infty$-ideal, and so of the form $\mathcal{K}(A)$ for some $A \in D_{2}$.

Proof OF TheOREMS 8 AND 9 . That every $\boldsymbol{\Pi}_{2}^{0} \sigma$-ideal which is not $\boldsymbol{\Pi}_{2}^{0}$ complete is in $D_{2}$ follows from Proposition 5(ii) and Corollary 3(ii), as in the proof of Theorem 7. Similarly if $I$ is in $D_{2}$ but not $D_{2}$-complete we use Proposition 5(i) and Corollary 3(i). It remains only to prove Theorem 9.

Let $I=\bigcup_{n} L_{n}$, where without loss of generality we can assume that $L_{n}$ are hereditary compact subsets of $\mathcal{K}(E)$. Put as usual $A_{I}=\{x \in E:\{x\} \in I\}$ and consider

$$
U_{0}=\bigcup\left\{U \text { open in } E: \mathcal{K}\left(A_{I} \cap U\right) \subseteq I\right\} .
$$

Since $I$ is an ideal, an easy compactness argument shows that $\mathcal{K}\left(A_{I} \cap U_{0}\right) \subseteq I$, i.e. $U_{0}$ is the largest open set $U$ with $\mathcal{K}\left(A_{I} \cap U\right) \subseteq I$. We want to prove that actually $\mathcal{K}\left(A_{I} \cap U_{0}\right)=I$. For that it is enough to check that $I \subseteq \mathcal{K}\left(U_{0}\right)$. If not, then $I^{\prime}=I-\mathcal{K}\left(U_{0}\right) \neq \varnothing$. Since $I$ is $\Pi_{2}^{0}$, so is $I^{\prime}$, so since $I^{\prime} \subseteq \bigcup_{n} L_{n}$, we can find by the Baire category theorem an open set $V$ in $K(E)$ and some $n$ with $V \cap I^{\prime} \neq \varnothing$ and $V \cap I^{\prime} \subseteq L_{n}$. We may assume that for some $G_{0}, G_{1}, \ldots, G_{k}$ open in $E, V=\left\{K: K \subseteq G_{0} \& K \cap G_{i} \neq \varnothing, 1 \leq i \leq k\right\}$. Let $K_{0} \in V \cap I^{\prime}$, so that $K_{0} \subseteq G_{0}, K_{0} \subseteq A_{I}$ but $K_{0} \nsubseteq U_{0}$. So $G_{0} \cap\left(A_{I}-U_{0}\right) \neq \varnothing$, hence $\mathcal{K}\left(A_{I} \cap G_{0}\right) \nsubseteq I$ (else $G_{0} \subseteq U_{0}$ ). We will derive a contradiction to this.

Let $K_{0}^{\prime} \subseteq K_{0}$ be finite with $K_{0}^{\prime} \in V$, and $K_{0}^{\prime} \cap\left(E-U_{0}\right) \neq \varnothing$. Then $K_{0}^{\prime} \in V \cap I^{\prime}$. If now $K \subseteq A_{I} \cap G_{0}$ is finite, $K \cup K_{0}^{\prime} \subseteq A_{I}$ and thus $K \cup K_{0}^{\prime} \in I$ (being finite). Also $K \cup K_{0}^{\prime} \nsubseteq U_{0}$, so $K \cup K_{0}^{\prime} \in I^{\prime}$ and clearly $K \cup K_{0}^{\prime} \in V$. So $K \subseteq K \cup K_{0}^{\prime} \in L_{n}$ and thus $K \in L_{n}$. So all finite subsets of $A_{I} \cap G_{0}$ are in $L_{n}$ and since $L_{n}$ is closed $\mathcal{K}\left(A_{I} \cap G_{0}\right) \subseteq L_{n} \subseteq I$, a contradiction.

In view of Theorem 8 all the $\Pi_{1}^{1} \sigma$-ideals of compact sets which are not of the form $\mathcal{K}(A)$ fall in exactly one of two categories:

(A) The "simple" ones, which are $\Pi_{2}^{0}$-complete. Typical examples are the nowhere dense compact sets or the $\mu$-measure 0 compact sets for any continuous finite measure $\mu$, on any perfect compact space $E$.

(B) The "complicated" ones, which are $\boldsymbol{\Pi}_{1}^{1}$-complete. Typical examples are the countable compact sets in a perfect compact space $E$ or the compact sets of uniqueness in the circle $\mathbf{T}$.

To finish this section, let us consider the case of $\Sigma_{1}^{1} \sigma$-ideals. It follows easily from the proof of Theorems 4 and 7 , that if we use the determinacy of $\Sigma_{1}^{1}$ games, the $\Sigma_{1}^{1} \sigma$-ideals are actually $\Pi_{2}^{0}$. This indeed can be proved without this additional assumption, using as a key step a lemma of Saint Raymond [StR1]. (This was 
the main lemma used by him to prove that $\mathcal{K}(A) \in \Sigma_{1}^{1} \Rightarrow A \in \mathbf{\Pi}_{2}^{0}$. Actually the hypotheses of the lemma in [StR1] are a bit stronger than the ones we use here, but one can easily check that the proof goes through with the weaker hypotheses given below.)

LEMMA 10 (SAINT RAYMOND [StR1]). Let $E$ be compact metrizable, and $A \subseteq E$ a $\Sigma_{1}^{1}$ set. Let $P$ be Polish and $\varphi: P \rightarrow A$ a continuous surjection such that for any compact countable $K \subseteq A$ there is compact $L \subseteq P$ with $\varphi^{\prime \prime}(L)=K$. Then $A$ is Polish, i.e. $\Pi_{2}^{0}$ in $E$.

We now have

THEOREM 11. Let I be a $\Sigma_{1}^{1} \sigma$-ideal of compact sets in some compact metrizable space $E$. Then $I$ is actually $\Pi_{2}^{0}$.

Proof. Consider

$$
J \subseteq \mathcal{K}(\mathcal{K}(E)), \quad \text { given by } L \in J \Leftrightarrow \bigcup L \in I .
$$

Since $I$ is $\Sigma_{1}^{1}$, so is $J$. So let $X$ be Polish and $f: X \rightarrow J$ a continuous surjection. Define $P \subseteq X \times K(E)$ by

$$
(x, K) \in P \Leftrightarrow K \in f(x) .
$$

Then $P$ is closed in $X \times K(E)$, so is Polish. Let $\varphi: P \rightarrow \mathcal{K}(E)$ be given by $\varphi(x, K)=$ $K$. Then $\varphi$ is continuous and we will check that it satisfies the hypotheses of Saint Raymond's lemma with $\varphi^{\prime \prime}(P)=I$. It then follows that $I$ is $\Pi_{2}^{0}$.

So first let $K \in I$. Then $\{K\} \in J$, so for some $x \in X,(x, K) \in P$ and thus $\varphi^{\prime \prime}(P) \supseteq I$. Conversely, if $(x, K) \in P$ then $K \in f(x) \in J$, hence $K \subseteq \bigcup f(x) \in I$, thus $K \in I$. So $\varphi^{\prime \prime}(P)=I$. Finally, if $L$ is a countable compact subset of $I$, then $\bigcup L \in I$, since $I$ is a $\sigma$-ideal, so $L \in J$ and thus let $x_{L} \in X$ be such that $f\left(x_{L}\right)=L$. Put $L^{\prime}=\left\{\left(x_{L}, K\right): K \in L\right\}=\left\{x_{L}\right\} \times L$. Then $L^{\prime}$ is a compact subset of $P$, and $\varphi^{\prime \prime}\left(L^{\prime}\right)=L$.

\section{Bases for $\sigma$-ideals of compact sets.}

2.1 The concept of basis. Let $I$ be a $\sigma$-ideal of compact sets in a compact metrizable space. A set $B \subseteq I$ is a basis for $I$ if $I$ is the $\sigma$-ideal generated by $B$, i.e. if for each $K \in I$ there is a sequence $\left\{K_{n}\right\}, K_{n} \in B$ with $K \subseteq \bigcup_{n} K_{n}$. If $B$ is hereditary this is equivalent to $I=B_{\sigma}$. We say that $I$ admits a $\Gamma$-basis if such a basis $B$ can be found in the class $\Gamma$. We will be mainly interested in the problem of existence of Borel bases for $\Pi_{1}^{1} \sigma$-ideals.

First an easy proposition.

Proposition 1. Let I be $a \Pi_{1}^{1} \sigma$-ideal of compact sets in some compact metrizable space. Then the following are equivalent:

(i) I admits a $\boldsymbol{\Sigma}_{1}^{1}$-basis;

(ii) I admits a Borel basis;

(iii) I admits a hereditary Borel basis.

Proof. Clearly (iii) $\Rightarrow$ (ii) $\Rightarrow$ (i). Let now $B_{0}$ be a $\Sigma_{1}^{1}$-basis. By separation find Borel $C_{0}$ with $B_{0} \subseteq C_{0} \subseteq I$. Let $B_{1}$ be the hereditary closure of $C_{0}$. Then $B_{1} \in \Sigma_{1}^{1}$ and $C_{0} \subseteq B_{1} \subseteq I$, hence there is Borel $C_{1}$ with $B_{1} \subseteq C_{1} \subseteq I$, etc. 
So inductively we define $B_{n} \subseteq C_{n} \subseteq B_{n+1}, C_{n}$ Borel and $B_{n+1}$ hereditary. Let $B=\bigcup_{n} B_{n}=\bigcup_{n} C_{n}$. Then $B$ is Borel, hereditary and a basis since $B_{0} \subseteq B$.

Proposition 1 has the following converse:

If a $\sigma$-ideal I admits a Borel, in fact even a $\Pi_{1}^{1}$ hereditary basis, then it is $\Pi_{1}^{1}$.

This follows from work of Cenzer and Mauldin, characterizing hereditary $\Pi_{1}^{1}$ subsets of $\mathcal{K}(E)$ as those sets $B$ for which there is a $\Pi_{1}^{1}$ set $T \subseteq E^{\omega}$ with $K \in B \Leftrightarrow$ $K^{\omega} \subseteq T$ (see $[\mathbf{C}-\mathbf{M}$ or $\mathbf{L 1}]$ ), and from work of Dellacherie, Hillard and Louveau $[$ Hi, L1]. An explicit proof of the result above - together with some generalizations can be found in [L1, Chapter 3, pp. 48-54].

However, one cannot drop the hypothesis that $B$ is hereditary: Let $A \subseteq E$ be a true $\Sigma_{1}^{1}$ set, and let

$$
I=\mathcal{K}_{\omega}(A)=\{K \in \mathcal{K}(E): K \text { is countable } \& K \subseteq A\} .
$$

Clearly $I$ admits the (hereditary) $\boldsymbol{\Sigma}_{1}^{1}$-basis $B=\{\varnothing\} \cup\{\{x\}: x \in A\}$, and we will see in the next subsection that this implies that $A$ admits a $\Pi_{2}^{0}$-basis. But $I$ is not $\Pi_{1}^{1}$, and so $I$ cannot have a hereditary Borel basis (this can be also seen directly as follows: If $C \subseteq I$ is hereditary Borel, $\{x \in E:\{x\} \in C\}$ is a Borel subset of $A$, so $C$ does not generate $I$ ).

Using similar ideas, one gets counterexamples to various possible conjectures, showing in particular (in combination with the results of 2.2) that the notions of basis and hereditary basis are quite different.

Let first $A$ be a true $\Pi_{1}^{1}$ set. Then $I=\mathcal{K}_{\omega}(A)$ is the simplest example of a $\Pi_{1}^{1} \sigma$-ideal with no Borel basis.

Let now $A$ be a Borel set. Then $I=\mathcal{K}_{\omega}(A)$ is $\Pi_{1}^{1}$ and admits a Borel basis, e.g. $B=\{\varnothing\} \cup\{\{x\}: x \in A\}$, but any hereditary Borel basis $C$ must be of Borel complexity at least that of $A$, since $x \in A \Leftrightarrow\{x\} \in C$. So the complexity of hereditary Borel bases can be arbitrarily high in the Borel hierarchy. This should be compared with the result in 2.2 showing that there is always a $\Pi_{2}^{0}$-basis (if there is a Borel one).

We will see now that the problem of the existence of a Borel basis is equivalent to a classification problem. by

For a $\sigma$-ideal $I$ of compact sets in $E$, let $I_{L}$ (the "local" version of $I$ ) be defined

$$
K \in I_{L} \Leftrightarrow \exists U \text { open in } E(K \cap U \neq \varnothing \& K \cap \bar{U} \in I) .
$$

For example, let $I=\mathcal{K}_{\omega}\left(2^{\omega}\right) \subseteq \mathcal{K}\left(2^{\omega}\right)$ be the $\sigma$-ideal of countable compact sets in $2^{\omega}$. It is well known that $I$ is $\Pi_{1}^{1}$-complete. (Here is a simple proof, based on Theorem 1.7: Let $\varphi: 2^{\omega} \rightarrow \mathcal{K}\left(2^{\omega}\right)$ be defined by $\varphi(\alpha)=\left\{\beta \in 2^{\omega}: \forall n(\alpha(n)=0 \Rightarrow\right.$ $\beta(n)=0\}$. Then $\varphi$ is continuous and $\varphi^{-1}[I]=\mathbf{Q}$, so $I$ is not $\Pi_{2}^{0}$.) Now $\mathcal{K}(E)-I_{L}$ consists of exactly the perfect compact subsets of $2^{\omega}$, which is a $\Pi_{2}^{0}$ set.

We have now

THEOREM 2. Let I be a $\Pi_{1}^{1} \sigma$-ideal of compact sets in a compact metrizable space $E$. Then the following are equivalent:

(i) I admits a Borel basis;

(ii) $I_{L}$ is Borel.

PROOF. If $I$ admits a hereditary Borel basis $B$ then by a Baire category argument we have

$$
K \in I_{L} \Leftrightarrow \exists U \text { open in } E(K \cap U \neq \varnothing \& K \cap \bar{U} \in B),
$$

so $I_{L}$ is Borel. 
Conversely if $I_{L}$ is Borel, let $\left\{U_{n}\right\}$ be a basis for open sets for $E$, and for each $n$ let $C_{n}=\left\{K: K \cap U_{n} \neq \varnothing \& K \cap \bar{U}_{n} \in I\right\}$. Then $C_{n} \in \Pi_{1}^{1}$ and $I_{L} \subseteq \bigcup_{n} C_{n}$. By Novikov's theorem there is a Borel function $\varphi: I_{L} \rightarrow \omega$ such that for $K \in$ $I_{L}, K \cap U_{\varphi(K)} \neq \varnothing \& K \cap \bar{U}_{\varphi(K)} \in I$. Let $B=\left\{K \cap \bar{U}_{\varphi(K)}: K \in I_{L}\right\}$. Then $B$ is $\Sigma_{1}^{1}$ and $B \subseteq I$, so it is enough to show $B$ is a basis. So let $K \in I$ and put

$$
\begin{array}{r}
K^{\prime}=K-\bigcup\{U \text { basic open in } E: U \cap K \text { is covered by the union of a } \\
\text { sequence of elements of } B\} .
\end{array}
$$

If we show that $K^{\prime}=\varnothing$, we are done. But if $K^{\prime} \neq \varnothing$, then since $K^{\prime} \in I$ we have that $K^{\prime} \in I_{L}$. But then for $U=U_{\varphi\left(K^{\prime}\right)}$ we have that both $K \cap U$ is covered by the union of a sequence of elements in $B$ and $K^{\prime} \cap U \neq \varnothing$, a contradiction.

Note that by the preceding proof we can also add another equivalence, namely

(iii) There is a Borel set $A$ with $I \subseteq A \subseteq I_{L} \cup\{\varnothing\}$.

$2.2 \Pi_{2}^{0}$-bases. We can view the following result as an analog of Theorem 1.11 for bases.

THEOREM 3. Let I be a $\sigma$-ideal of compact sets in a compact metrizable space E. If I has a $\boldsymbol{\Sigma}_{1}^{1}$-basis, then it actually has a $\mathbf{\Pi}_{2}^{0}$-basis.

ProOF. We can of course assume that $I$ has a hereditary $\Sigma_{1}^{1}$-basis $B$. We distinguish two cases.

Case 1. Every set in $I$ is countable. Then $I=\mathcal{K}_{\omega}\left(A_{I}\right)$, where $A_{I}=\{x:\{x\} \in$ $I\}=\{x:\{x\} \in B\}$ is $\Sigma_{1}^{1}$ in $E$. We have now two subcases:

(a) If $A_{I}$ is uncountable, let $K_{0}$ be a copy of $2^{\omega}$ inside $A_{I}$ and let $P \subseteq E \times K_{0}$ be a $\Pi_{2}^{0}$ set projecting to $A_{I}-K_{0}$. Then $A=\left\{\{x\}: x \in K_{0}\right\} \cup\{\{x, y\}: x \in$ $\left.A_{I}-K_{0} \&(x, y) \in P\right\}$ is a $\Pi_{2}^{0}$-basis for $I$.

(b) If $A_{I}$ is countable, say $A_{I}=\left\{x_{n}: n \in \omega\right\}$, let $A$ consist of $\varnothing,\left\{x_{0} \cdots x_{n}\right\}, n \in$ $\omega$. One checks that $\bar{A}-A=\left\{\bar{A}_{I}\right\}$, hence $A$ is $\Pi_{2}^{0}$ (in fact $D_{2}$ ) and clearly a basis for $I$.

Case 2. I contains some compact perfect set $K_{0}$. Choose first a sequence $\left\{V_{n}\right\}$ of open sets with $\bar{V}_{n} \subseteq V_{n+1}$ and $\bigcup_{n} V_{n}=E-K_{0}$. Then choose open sets $U_{n}$ with $K_{0} \cap U_{n} \neq \varnothing, U_{n} \cap V_{n}=\varnothing$ and $U_{n} \cap U_{m}=\varnothing$ if $n \neq m$.

Now let $B_{n}=B \cap \mathcal{K}\left(\bar{V}_{n}\right)$. Since $B_{n}$ is $\Sigma_{1}^{1}$ in $\mathcal{K}(E)$ and $\mathcal{K}\left(U_{n} \cap K_{0}\right)$ contains a copy of $2^{\omega}$, there exists a $\boldsymbol{\Pi}_{2}^{0}$ set

$$
P_{n} \subseteq \mathcal{K}(E) \times\left(\mathcal{K}\left(U_{n} \cap K_{0}\right)-\{\varnothing\}\right)
$$

with $\pi P_{n}=B_{n}$. Put

$$
\begin{aligned}
& A=\left\{K_{0}\right\} \cup\{K \in \mathcal{K}(E): \exists n\left(K \subseteq U_{n} \cup V_{n}\right) \\
& \& \forall n\left[K \subseteq U_{n} \cup V_{n} \Rightarrow K \subseteq K_{0} \cup \bar{V}_{n}\right. \\
&\left.\left.\quad \&\left(K \cap \bar{V}_{n}, K \cap K_{0}\right) \in P_{n}\right]\right\} .
\end{aligned}
$$

First $A$ is a $\Pi_{2}^{0}$ set: This is because the function $K \mapsto\left(K \cap \bar{V}_{n}, K \cap K_{0}\right)$ from $\mathcal{K}\left(K_{0} \cup \bar{V}_{n}\right)$ into $\mathcal{K}(E) \times \mathcal{K}(E)$ is continuous, since $\bar{V}_{n}$ and $K_{0}$ are clopen in $K_{0} \cup \bar{V}_{n}$.

Also $A \subseteq I$ : Indeed if $K \in A$, either $K=K_{0} \in I$ or for some $n K=\left(K \cap \bar{V}_{n}\right) \cup$ $\left(K \cap K_{0}\right)$ and $K \cap \bar{V}_{n} \in \pi P_{n}=B_{n}$, so $K \cap \bar{V}_{n} \in I$ and then $K \in I$. 
Finally we check that $A$ is a basis for $I$ : Let $K \in B$. Then for each $n K \cap$ $\bar{V}_{n} \in B_{n+1}$, so for some $K_{n}^{\prime} \subseteq U_{n+1} \cap K_{0}, K_{n}^{\prime} \neq \varnothing,\left(K \cap \bar{V}_{n}, K_{n}^{\prime}\right) \in P_{n+1}$. We claim that $K_{n}^{\prime \prime}=\left(K \cap \bar{V}_{n}\right) \cup K_{n}^{\prime}$ is in $A$, which completes the proof since $K=K_{0} \cup \bigcup_{n}\left(K \cap \bar{V}_{n}\right) \subseteq K_{0} \cup \bigcup_{n} K_{n}^{\prime \prime}$. Indeed, $K_{n}^{\prime \prime} \subseteq U_{n+1} \cup V_{n+1}$ and if $K_{n}^{\prime \prime} \subseteq U_{m} \cup V_{m}$, then clearly $m=n+1$ (recall that the $V_{m}$ 's are disjoint and $\left.\varnothing \neq K_{n}^{\prime} \subseteq U_{n+1}\right)$; so $K_{n}^{\prime \prime} \subseteq K_{0} \cup \bar{V}_{m}$ and $\left(K_{n}^{\prime \prime} \cap \bar{V}_{m}, K_{n}^{\prime \prime} \cap K_{0}\right)=\left(K \cap \bar{V}_{n}, K_{n}^{\prime}\right) \in P_{m}$, so we are done.

This result is best possible, because if $A \subseteq E$ is a true $\Pi_{2}^{0}$ set, then $\mathcal{K}(A)$ is $\Pi_{2}^{0}$ but cannot have a $\Sigma_{2}^{0}$-basis, since otherwise $A$ would have been $\Sigma_{2}^{0}$ also.

But if a $\sigma$-ideal has a $\Sigma_{2}^{0}$-basis then one has a further reduction.

THEOREM 4. Let I be a $\sigma$-ideal of compact sets in a compact metrizable space. If I has a $\boldsymbol{\Sigma}_{2}^{0}$-basis, it has actually a $D_{2}$-basis.

ProOF. First note that $I$ is $\Pi_{1}^{1}$ (by the remarks following Proposition 1) since $I$ has a hereditary $\Sigma_{2}^{0}$ - basis $B$. We have again two cases:

Case 1. Ever $K \in I$ is finite. Then $A_{I}=\{x \in E:\{x\} \in I\}$ must be discrete and so in $D_{2}$, and therefore $I=\mathcal{K}\left(A_{I}\right)$ is $D_{2}$ itself.

Case 2. Some $K \in I$ is infinite. Then there is $K_{0} \in I$ homeomorphic to $\omega+1$, say $K_{0}=\left\{x_{n}: n \in \omega\right\} \cup\left\{x_{\omega}\right\}$. Let $U_{n}=E-\left(\left\{x_{m}: m \geq n\right\} \cup\left\{x_{\omega}\right\}\right)$. Then $U_{n}$ is open and $U_{0}=E-K_{0}$. Let $B^{\prime}=B \cap \mathcal{K}\left(U_{0}\right)$. Then $B^{\prime}$ is $\Sigma_{2}^{0}$ in $\mathcal{K}(E)$, so let $B^{\prime}=\bigcup_{n} L_{n}$, with $L_{n}$ closed in $\mathcal{K}(E)$, and $L_{n} \subseteq L_{n+1}$. Put

$$
A=\left\{K: K=\left\{x_{\omega}\right\} \text { or } \exists n \exists K^{\prime} \in L_{n}\left[K=K^{\prime} \cup\left\{x_{0} \cdots x_{n}\right\}\right]\right\} \text {. }
$$

Clearly $A \subseteq I$ and $A$ is a basis for $I$.

We prove that $A \in D_{2}$ : First note that $x_{0}$ is contained in every element of $A$ except $\left\{x_{\omega}\right\}$ so $\left\{x_{\omega}\right\}$ is an isolated point in $A$. Thus it is enough to show $A^{\prime}=A-\left\{\left\{x_{\omega}\right\}\right\}$ is in $D_{2}$. For that let

$$
L_{n}^{\prime}=\bigcup_{p<n}\left\{K \cup\left\{x_{0} \cdots x_{p}\right\}: K \in L_{p}\right\}
$$

so that $L_{n}^{\prime}$ is closed (in $\mathcal{K}(E)$ ). Then notice that

$$
K \in A^{\prime} \Leftrightarrow \exists n>0\left(K \subseteq U_{n}\right) \& \forall n>0\left(K \subseteq U_{n} \Rightarrow K \in L_{n}^{\prime}\right),
$$

so $A^{\prime}$ is $D_{2}$.

Note again that this is best possible: If $A \subseteq E$ is a true $D_{2}$ set, $\mathcal{K}(A)$ is a $D_{2}$ $\sigma$-ideal with no $\check{D}_{2}$-basis.

Finally we have

THEOREM 5. Suppose I is a $\sigma$-ideal of compact sets in some compact metrizable space. If I has a $\check{D}_{2}$-basis, it has actually a $\Delta\left(\check{D}_{2}\right)=\Delta\left(D_{2}\right)$-basis.

Proof. Let $B_{1}$ in $\check{D}_{2}$ be a basis for $I$. Say $B_{1}=F \cup U_{1}$, where $F$ is closed and $U_{1}$ is open. We can assume that $F$ is hereditary. Moreover $U_{1}$ is the union of a countable sequence $\bigcup_{n} V_{n}$, where $\varnothing \neq V_{n}=\left\{K \in \mathcal{K}(E): K \subseteq G^{(n)} \& K \cap G_{1}^{(n)} \neq\right.$ $\left.\varnothing \& \cdots \& K \cap G_{k_{n}}^{(n)} \neq \varnothing\right\}$, with $G_{i}^{(n)}$ open in $E$.

Let $V_{n}^{\prime}=\mathcal{K}\left(G^{(n)}\right)$. Then $V_{n} \subseteq V_{n}^{\prime} \subseteq I$, so if $U=\bigcup_{n} V_{n}^{\prime}$, then $B=F \cup U$ is still a basis and $F, U$ are hereditary, $F$ is closed and $U$ is open. 
Let now $V=A_{U}=\{x:\{x\} \in U\}$. Then $V$ is open in $E$ and for each $x \in V$ there is $n$ with $x \in G^{(n)}$ and $\mathcal{K}\left(G^{(n)}\right) \subseteq U$. So $U \subseteq \mathcal{K}(V) \subseteq I$. Let $L=E-V$, and $B^{\prime}=B \cap \mathcal{K}(L)=F \cap \mathcal{K}(L)$. If $V=\varnothing$, then $B^{\prime}$ is a closed basis for $I$. If $V \neq \varnothing$, choose $V^{\prime}$ open, $V^{\prime} \neq \varnothing$, with $\bar{V}^{\prime} \subseteq V$ and let $A=B^{\prime} \cup\left\{K: K \subseteq V \& K \cap V^{\prime} \neq \varnothing\right\}$. Then $A$ is a basis for $I$. Now

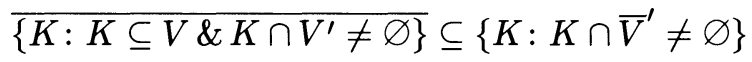

so the closure of $\left\{K: K \subseteq V \& K \cap V^{\prime} \neq \varnothing\right\}$ is disjoint from $B^{\prime}$, thus $A \in \Delta\left(D_{2}\right)$ and we are done.

As usual this is best possible: If $A$ is a $\Delta\left(D_{2}\right)$ set in $E$ which is neither closed nor open, then $\mathcal{K}_{\omega}(A)$ has a $\Delta\left(D_{2}\right)$-basis but not an open or closed one.

If $I$ has an open basis then an argument as in the preceding proof shows that $I=\mathcal{K}\left(A_{I}\right)$ is itself open. But on the other hand $\mathcal{K}_{\omega}\left(2^{\omega}\right)$ has a closed basis (i.e. $\{\varnothing\} \cup$ $\left.\left\{\{x\}: x \in 2^{\omega}\right\}\right)$, but is complete $\Pi_{1}^{1}$.

We conclude with a few questions:

Q1. What can be said about ideals with closed bases? (They should be somehow simple.)

Q2. What is the exact maximum complexity of $\sigma$-ideals with $\Sigma_{1}^{1}\left(\therefore \Pi_{2}^{0}\right)$ bases? (They must all be $\Sigma_{2}^{1}$.)

Q3. Say that a $\Pi_{2}^{0}$-basis $B$ of a $\sigma$-ideal $I$ is homogeneous if for every $K \in$ $\mathcal{K}(E), B_{K}=\{K \cap L: L \in B\}$ is $\Pi_{2}^{0}$. Which $\sigma$-ideals admit homogeneous $\Pi_{2}^{0}$ bases?

2.3. Which $\Pi_{1}^{1} \sigma$-ideals have no Borel basis? The search for $\sigma$-ideals with no Borel basis seems quite hard. Ordinary examples of $\Pi_{1}^{1} \sigma$-ideals like the nowhere dense compact sets, the zero sets for a Radon measure, a Hausdorff measure or a capacity, are all $\Pi_{2}^{0} \sigma$-ideals, while some of the standard examples of true $\Pi_{1}^{1} \sigma$-ideals such as $\mathcal{K}(\mathbf{Q})$ or $\mathcal{K}_{\omega}\left(2^{\omega}\right)$ have natural Borel bases. Of course an example is $\mathcal{K}_{\omega}(A)$ (or $\mathcal{K}(A)$ ) for some true $\Pi_{1}^{1}$ set $A$, but one is looking for more interesting examples. For instance as we said earlier it is not known if the $\sigma$-ideal of compact sets of uniqueness has a Borel basis. (The nonexistence of a Borel basis here would have interesting implications in the theory of sets of uniqueness.)

Here is however an example of an interesting $\Pi_{1}^{1} \sigma$-ideal with no Borel basis.

Proposition 6. Let $E=2^{\omega}$ and let $E^{\#}=\{\mu: \mu(E)=1, \mu \geq 0\}$ with the weak*-topology. To each $K \in \mathcal{K}\left(E^{\#}\right)$ associate the capacity $\gamma_{K}$ defined by

$$
\gamma_{K}(F)=\sup \{\mu(F): \mu \in K\} .
$$

Let $I=\left\{K \in \mathcal{K}\left(E^{\#}\right): \gamma_{K}\right.$ is thin $\}$. Then $I$ is a $\Pi_{1}^{1} \sigma$-ideal with no Borel basis.

(Recall that a capacity is thin if there is no uncountable family of pairwise disjoint compact sets of positive capacity.)

Proof. Let $\varphi: \mathcal{K}(E) \rightarrow \mathcal{K}\left(E^{\#}\right)$ be defined by $\varphi(K)=K^{\#}=\left\{\mu \in E^{\#}: \mu(K)\right.$ $=1\}$. Then $\varphi$ is continuous, and we claim that $\varphi^{-1}[I]=\varphi^{-1}\left[I_{L} \cup\{\varnothing\}\right]=K_{\omega}(E)$, which implies that $I_{L}$ is not Borel and by Theorem 2 finishes the proof. If $K \in \mathcal{K}(E)$ is countable then $\gamma_{K}$ is clearly thin, so $K_{\omega}(E) \subseteq \varphi^{-1}[I] \subseteq \varphi^{-1}\left[I_{L} \cup\{\varnothing\}\right]$. Assume now $K$ is uncountable, towards showing that $K^{\#} \notin I_{L} \cup\{\varnothing\}$. Let $U$ be open in $E^{\#}$ such that $K^{\#} \cap U \neq \varnothing$. We want to prove that $\gamma_{K^{\#} \cap \bar{U}}$ is not thin. Let $K_{0} \subseteq K$ be a copy of $2^{\omega}$. If $\mu_{0} \in K^{\#} \cap U$ then by the definition of the weak*-topology there is 
$\varepsilon>0$ with $\forall x \in K_{0}\left[\left(\varepsilon \cdot \delta_{x}+(1-\varepsilon) \cdot \mu_{0}\right) \in K^{\#} \cap U\right]$, where $\delta_{x}$ is the Dirac measure at $x$. But then $\left\{\{x\}: x \in K_{0}\right\}$ is an uncountable family demonstrating that $\gamma_{K^{\#} \cap \bar{U}}$ is not thin.

We proceed now to establish a sufficient criterion for nonexistence of Borel bases for $\Pi_{1}^{1} \sigma$-ideals. As application we will solve completely the problem of when the ideal $\mathcal{K}(A), A \in \Pi_{1}^{1}$ has a Borel basis and we will give also another interesting example of a $\Pi_{1}^{1} \sigma$-ideal with no Borel basis. This criterion will look a bit technical at first sight but we will give some motivation immediately after stating it.

LEMMA 7 (A sufficient criterion for nonexistence of Borel bases). Let $I$ be a $\Pi_{1}^{1} \sigma$-ideal in $\mathcal{K}(E), E$ compact metrizable and let $\left\{J_{n}\right\}, J_{n} \subseteq \mathcal{K}(E)$ and $D \subseteq E$ satisfy:

(a) $J_{n}$ is nonempty hereditary open in $\mathcal{K}(E)$, and $\left[K \in J_{n} \& x \in D \Rightarrow K \cup\right.$ $\left.\{x\} \in J_{n}\right]$,

(b) Let I $=\left\{X \subseteq E: \forall K \in \mathcal{K}(X) \forall n\left(K \in J_{n}\right)\right\}$. If $\left\{K_{n}\right\}$ is a sequence of sets in $I, H \in I$ is $a G_{\delta}$ and $K=H \cup \bigcup_{n} K_{n}$ is compact, then $K \in I$.

Then if $I$ is true $\Pi_{1}^{1}$ and $D=E$, or $I \cap \mathcal{K}(U)$ is true $\Pi_{1}^{1}$ for all open nonempty $U \subseteq E$ and $D$ is dense in $E$, the $\sigma$-ideal $I$ has no Borel basis.

Let us mention an immediate corollary which was an original motivation for this kind of criterion, and whose proof illustrates also the meaning of the hypotheses above.

COROLLARY 8. Let I be a true $\Pi_{1}^{1} \sigma$-ideal of compact sets on a compact metrizable space $E$. Let $\gamma$ be a capacity on $E$ such that $\gamma(K \cup\{x\})=\gamma(K)$ for $K \in \mathcal{K}(E)$ and $x \in E$. Let $\tilde{I}_{\gamma}$ be the class of null sets for the capacity $\gamma$. Assume that if $K_{n} \in I, \forall n, H$ is $a G_{\delta}, H \in \tilde{I}_{\gamma}$ and $K=H \cup \bigcup_{n} K_{n}$ is compact then $K \in I$. Then I has no Borel basis.

In particular (using the terminology of 3.2) if $I$ is a true $\Pi_{1}^{1}$ calibrated $\sigma$-ideal [i.e. $K \doteq H \cup \bigcup_{n} K_{n}$, where $K \in \mathcal{K}(E), K_{n} \in I, H \in G_{\delta}$ and $\forall L \in \mathcal{K}(H)(L \in I)$, implies $K \in I]$, then $I_{\gamma}=\tilde{I}_{\gamma} \cap \mathcal{K}(E) \subseteq I \Rightarrow I$ has no Borel basis. In other words every calibrated true $\Pi_{1}^{1} \sigma$-ideal which contains the compact zero sets of some capacity as above has no Borel basis.

If we call, for any capacity $\gamma$, a set $A \subseteq E \gamma$-thin if there is no uncountable family of pairwise disjoint sets in $\mathcal{K}(A)$ of positive capacity, then

$$
J_{\gamma}=\{K \in \mathcal{K}(E): K \text { is } \gamma \text {-thin }\}
$$

is a calibrated $\Pi_{1}^{1} \sigma$-ideal of compact sets (see for example Corollary 3.4). Thus, if $\gamma$ satisfies $\gamma(K \cup\{x\})=\gamma(K)$, since clearly $I_{\gamma} \subseteq J_{\gamma}$, it follows that

$$
J_{\gamma} \text { is Borel } \Leftrightarrow J_{\gamma} \text { has a Borel basis. }
$$

Sometimes the ideal $J_{\gamma}$ is Borel. For example the electrostatic capacity $\gamma_{0}$ has the strange property that $J_{\gamma_{0}}=I_{\gamma_{0}}$, so $J_{\gamma_{0}}$ is $\Pi_{2}^{0}$ in this case. But another natural capacity gives an example where $J_{\gamma}$ has no Borel basis. 
Proposition 9. Let $E=[0,1] \times[0,1]$, and let for $A \subseteq E, \gamma(A)=\mu(\pi A)$. Then $\gamma$ is a capacity with $\gamma(K \cup\{x\})=\gamma(K)$ for any $K \in \mathcal{K}(E), x \in E$ and

$$
\begin{aligned}
I & =J_{\gamma}=\{K \in \mathcal{K}(E): K \text { is } \gamma \text {-thin }\} \\
& =\left\{K \in \mathcal{K}(E): \mu\left\{x \in[0,1]: K_{x} \text { is uncountable }\right\}=0\right\}
\end{aligned}
$$

is a (translation and homotheties invariant) $\Pi_{1}^{1} \sigma$-ideal with no Borel basis.

Proof. It is enough to prove that $I$ is a true $\Pi_{1}^{1}$ set. But $\{K \subseteq[0,1]:[0,1] \times$ $\mathcal{K} \in I\}=\mathcal{K}_{\omega}([0,1])$, so $I$ cannot be Borel.

We prove now Corollary 8 (from the lemma):

Take $J_{n}=\{K \in \mathcal{K}(E): \gamma(K)<1 / n\}$, and $D=E$. Clearly (a) is satisfied, since $\gamma(K)=\inf _{U \supseteq K ; U \text { open }} \gamma(U)$. For (b) note that $H \in \boldsymbol{\Pi}_{2}^{0}, H \in I \Leftrightarrow H \in \tilde{I}_{\gamma}$ by the capacitability theorem for $\boldsymbol{\Pi}_{2}^{0}$ sets.

We finally give the

PROOF OF LEMMA 7. First let us notice that it is enough to prove the second case of this lemma. Because if $I$ is true $\Pi_{1}^{1}$ and $D=E$, let $U_{0}=\bigcup\{U$ open in $E: I \cap \mathcal{K}(U)$ is $\left.\Pi_{2}^{0}\right\}$. Then $I \cap \mathcal{K}\left(U_{0}\right)$ is $\Pi_{2}^{0}$, so $E^{\prime}=E-U_{0} \neq \varnothing$ by hypothesis. Working in $E^{\prime}, I^{\prime}=I \cap \mathcal{K}\left(E^{\prime}\right), J_{n}^{\prime}=J_{n} \cap \mathcal{K}\left(E^{\prime}\right)$ and $D^{\prime}=E^{\prime}$ satisfy the hypotheses of the second case, so $I^{\prime}$ has no Borel basis and thus neither has $I$.

So let us assume $I \cap \mathcal{K}(U)$ is true $\Pi_{1}^{1}$ for all nonempty $U \subseteq E$ and $D$ is dense in $E$. Let $B \subseteq I$ be Borel and hereditary. We want to prove that $B$ is not a basis for $I$.

First note that for each nonempty $U$ open in $E, I \cap \mathcal{K}(U) \neq B \cap \mathcal{K}(U)$, so there is compact $K_{U} \subseteq U, K_{U} \in I-B$. Also each $K \in I$ is nowhere dense (because if $\varnothing \neq U \subseteq K, I \cap \mathcal{K}(U)=\mathcal{K}(U)$ is open). Finally if $K$ is nowhere dense compact in $E, U \supseteq K$ is open and $D$ is dense in $E$ we can find a discrete sequence of points

$$
D(K, U)=\left\{x_{n}(K, U): n \in \omega\right\} \subseteq(D \cap U)-K
$$

such that $\overline{D(K, U)}=D(K, U) \cup K$.

We proceed now to construct for each $s \in \omega^{<\omega}$ a compact set $K_{s}$ and an open set $U_{s}$ satisfying the following:

(i) $U_{s} \neq \varnothing \& K_{s}=K_{U_{s}}$ (hence $K_{s} \in I-B$ ),

(ii) $n \neq m \Rightarrow \bar{U}_{s^{\wedge} n} \cap \bar{U}_{s^{\wedge} m}=\varnothing$,

(iii) $\bar{U}_{s^{\wedge} n} \subseteq U_{s} \& \bar{U}_{s^{\wedge} n} \cap K_{s}=\varnothing$,

(iv) $\operatorname{diam}\left(\bar{U}_{s^{\wedge} n}\right) \leq 2^{-|s|}$,

(v) $\overline{\bigcup_{n} U_{s^{\wedge} n}}=\left(\bigcup_{n} \bar{U}_{s^{\wedge} n}\right) \cup K_{s}$,

(vi) $K_{s} \subseteq \overline{U_{n} K_{s^{\wedge} n}}$

(vii) If $K$ is compact and $K \subseteq \bigcup_{|s|=n+1} U_{s}$, then $K \in J_{n}$.

Let $U_{\varnothing}=E, K_{\varnothing}=K_{U_{\varnothing}}$. Suppose we have constructed $U_{s}, K_{s}$ for $|s| \leq k$ satisfying (i)-(vi). For $|s|=k$, consider $D\left(K_{s}, U_{s}\right)$ and enumerate $\bigcup_{|s|=k} D\left(K_{s}, U_{s}\right)$ as $\left\{x_{n}: n \in \omega\right\}$. Since $x_{0} \in D$ we have by (a) that $\left\{x_{0}\right\} \in J_{k}$. But as $\left\{x_{0}\right\}=$ $\bigcap_{N} \bar{B}\left(x_{0}, 1 / N\right)$ and $J_{k}$ is open, one of the balls $\bar{B}\left(x_{0}, 1 / N\right) \in J_{k}$. If $x_{0}$ is the $n$th point of $D\left(K_{s}, U_{s}\right)$ i.e. $x_{0}=x_{n}\left(K_{s}, U_{s}\right)$ we choose $N=N_{0}$ large enough to have

$$
\operatorname{diam}\left(B\left(x_{0}, 1 / N_{0}\right)\right) \leq 2^{-|s|}=2^{-k}
$$

$\bar{B}\left(x_{0}, 1 / N_{0}\right) \cap K_{s}=\varnothing, \bar{B}\left(x_{0}, 1 / N_{0}\right) \subseteq U_{s}$ and $\bar{B}\left(x_{0}, 1 / N_{0}\right) \cap\left\{x_{n}: n \geq 1\right\}=\varnothing$. Let then $U_{s^{\wedge} n}=B\left(x_{0}, 1 / N_{0}\right)$. Now we look at $x_{1}$; say $x_{1}=x_{m}\left(K_{t}, U_{t}\right)$, with $|t|=k$. 
By (a)

$$
\bar{B}\left(x_{0}, 1 / N_{0}\right) \cup\left\{x_{1}\right\}=\bigcap_{N}\left(\bar{B}\left(x_{0}, 1 / N_{0}\right) \cup \bar{B}\left(x_{1}, 1 / N\right)\right)
$$

is in $J_{k}$, so we can find $N=N_{1}$ so that

$$
\begin{gathered}
\bar{B}\left(x_{0}, 1 / N_{0}\right) \cup \bar{B}\left(x_{1}, 1 / N_{1}\right) \in J_{k}, \quad \operatorname{diam}\left(B\left(x_{1}, 1 / N_{1}\right)\right) \leq 2^{-(k+1)}, \\
\bar{B}\left(x_{1}, 1 / N_{1}\right) \cap K_{t}=\varnothing, \quad \bar{B}\left(x_{1}, 1 / N_{1}\right) \subseteq U_{t}, \quad \bar{B}\left(x_{0}, 1 / N_{0}\right) \cap \bar{B}\left(x_{1}, 1 / N_{1}\right)=\varnothing,
\end{gathered}
$$

and

$$
\bar{B}\left(x_{1}, 1 / N_{1}\right) \cap\left\{x_{n}: n \geq 2\right\}=\varnothing .
$$

Let then $U_{t^{\wedge} m}=B\left(x_{1}, 1 / N_{1}\right)$. Continuing this way we construct sets $U_{s^{\wedge} n}$ for all $|s|=k, n \in \omega$ and then we let $K_{s^{\wedge} n}=K_{U_{s} n_{n}}$. Then (i)-(vi) are clearly satisfied. For (vii) note that if $K \subseteq \bigcup_{|s|=k+1} U_{s}$, then by compactness $K \in J_{k}$.

Let now $H=\bigcap_{n} \bigcup_{|s|=n} U_{s}, K=H \cup \bigcup_{s} K_{s}$. Clearly $H$ is a $\Pi_{2}^{0}$ set and every $L \in \mathcal{K}(E), L \subseteq H$ is in $\bigcap_{n} J_{n}$ by (vii). Also $K_{s} \in I$, for all $s$. We claim now that $K$ is compact, so that by (b) $K \in I$, but also that $K$ cannot be covered by a sequence of elements of $B$, which leads to a contradiction and finishes the proof.

$K$ is compact: In fact we have $K=\bigcap_{n} \overline{\bigcup_{|s|=n} U_{s}}$. The inclusion $\subseteq$ is easy by (vi). If now $x \in \bigcap_{n} \overline{\bigcup_{|s|=n} \bigcup_{s}}$ but $x \notin \bigcup_{s} K_{s}$ then by (v) for each $n, x \in \bar{U}_{s}$ for some $|s|=n$, and by (ii), (iii) $x \in \bigcap_{n} \bigcup_{|s|=n} U_{s}=H$.

$K$ cannot be covered by a sequence of elements in $B$ : Suppose not. Then by the Baire category theorem there is $U_{0}$ open in $E$ with $U_{0} \cap K \neq \varnothing$ and $\bar{U}_{0} \cap K \in B$. Let $x \in U_{0} \cap K$. If $x \in H$ then for some unique $\alpha \in \omega^{\omega},\{x\}=\bigcap_{n} \bar{U}_{\alpha \nmid n}$ by (ii), (iii) and (iv), hence for some $n_{0}, \bar{U}_{\alpha \nmid n_{0}} \subseteq U_{0}$ and $K_{\alpha \nmid n_{0}} \subseteq U_{0} \cap K$, so $K_{\alpha \nmid n_{0}} \in B$, a contradiction. If finally $x \in K_{s}$, there is a sequence of sets $\left\{\bar{S}_{s^{\wedge}} n_{k}: k \in \omega\right\}$ converging to $\{x\}$, so for some $p, \bar{U}_{s^{\wedge} p} \subseteq U_{0}$, hence $K_{s^{\wedge} p} \subseteq U_{0} \cap K$, which again gives a contradiction, and we are done.

We will apply now Lemma 7 to characterize the $\Pi_{1}^{1}$ sets $A$ for which $\mathcal{K}(A)$ has a Borel basis.

THEOREM 10. Let $E$ be compact metrizable and $A \subseteq E$ be $\mathbf{\Pi}_{1}^{1}$. Then $\mathcal{K}(A)$ admits a Borel (hence $\boldsymbol{\Pi}_{2}^{0}$ ) basis iff $A$ is the difference of two $\boldsymbol{\Pi}_{2}^{0}$ sets.

Proof. If $A=H \cap \bigcup_{n} K_{n}$, where $H \in \Pi_{2}^{0}$ and $K_{n} \in \mathcal{K}(E)$, let $B=$ $\bigcup_{n}\left\{K: K \subseteq H \cap K_{n}\right\}$. Then $B$ is Borel (in fact the difference of two $\Pi_{2}^{0}$ sets), and clearly is a basis for $\mathcal{K}(A)$. Suppose now $\mathcal{K}(A)$ admits a Borel basis. Then $A$ is Borel. If $A$ is not the difference of two $\Pi_{2}^{0}$ sets then by Saint Raymond's theorem (1.6) there is a copy $F$ of $2^{\omega} \times 2^{\omega}$ inside $E$ with $A \cap F$ the corresponding copy of $A_{3}=\{(\alpha, \beta): \alpha \notin \mathbf{Q}$ or $\beta \in \mathbf{Q}\}$. Since $\mathcal{K}(A)$ is assumed to have a Borel basis so does $\mathcal{K}\left(A_{3}\right)$. So it is enough to show that $I=\mathcal{K}\left(A_{3}\right)$ has no Borel basis.

Let $\left\{U_{n}\right\}$ be a decreasing sequence of open sets in $2^{\omega} \times 2^{\omega}$ with $D=\bigcap_{n} U_{n}=$ $\{(\alpha, \beta): \alpha \notin \mathbf{Q}\}$. Let $J_{n}=\mathcal{K}\left(U_{n}\right)$. We claim that $I,\left\{J_{n}\right\}$ and $D$ satisfy the hypotheses of Lemma 7 and this will complete the proof.

First $D$ is clearly dense in $2^{\omega} \times 2^{\omega}$. Also $J_{n}$ is hereditary open and if $K \in J_{n}$, i.e. $K \subseteq U_{n}$ and $x \in D$, then $K \cup\{x\} \subseteq U_{n}$, so (a) is satisfied.

If now $H$ is $\Pi_{2}^{0}$ for each $K \in \mathcal{K}(H), K \in J_{n}$ for all $n$ then $H \subseteq D$ and so if $L=H \cup \bigcup_{n} K_{n}$ is compact with $K_{n} \in I$ then $L \subseteq D \cup A_{3}=A_{3}$, so $L \in I$. 
Finally if $U$ is nonempty and basic in $2^{\omega} \times 2^{\omega}$, then $U \cap A_{3}$ is homeomorphic to $A_{3}$, hence not $\Pi_{2}^{0}$, so $I \cap \mathcal{K}(U)=\mathcal{K}\left(U \cap A_{3}\right)$ is complete $\Pi_{1}^{1}$ by 1.7 (or merely the Christensen-Saint Raymond Theorem). So Lemma 7 applies and $K\left(A_{3}\right)$ has no Borel basis.

We finally study the problem of $\Sigma_{2}^{0}$-bases. We start with the following simple

LEMMA 11. Let I be a $\sigma$-ideal of compact sets in a compact metrizable space E. If I contains only nowhere dense sets then

$$
I-\{\varnothing\} \text { is nonmeager in } \mathcal{K}(E) \Rightarrow I \text { has no } \Sigma_{2}^{0} \text {-basis. }
$$

Proof. Assume $I$ has a $\Sigma_{2}^{0}$-basis and consider $I_{L}$. By the proof of Theorem 2 $I_{L}$ is $\Sigma_{2}^{0}$ and clearly $I-\{\varnothing\} \subseteq I_{L}$. But $\mathcal{K}(E)-I_{L}$ is dense, since it contains all sets of the form $\bar{G}_{1} \cup \cdots \cup \bar{G}_{n}$, where $\bar{G}_{i}$ are basic open. So $I_{L}$ is meager and $I-\{\varnothing\}$.

Thus for a $\sigma$-ideal $I$ which contains all singletons and only nowhere dense sets it follows that

(i) $I-\{\varnothing\}$ is meager $\Rightarrow I$ is complete $\Pi_{1}^{1}$.

(ii) $I-\{\varnothing\}$ is nonmeager $\Rightarrow I$ has no $\Sigma_{2}^{0}$-basis.

(For (i) just note that $I-\{\varnothing\}$ contains all singletons, therefore is dense.)

We can also use Lemma 11 to see for example the following

COROLlary 12. The $\sigma$-ideal of sets of uniqueness (in $\mathbf{T}$ ) does not have a $\mathbf{\Sigma}_{2}^{0}$ basis.

PROOF. It is enough to show that this ideal is comeager in $K(\mathbf{T})$. Here are two different arguments.

ARgument 1 (DUe TO SAINT RAYMOND). We use the notation of [K-S]. Let

$$
\begin{array}{r}
F_{N}=\{K \in \mathcal{K}(\mathbf{T}): \exists S \in P M(K)(\forall n(|\hat{S}(n)| \leq 1) \\
\left.\&|\hat{S}(0)| \geq \frac{2}{3} \& \forall|n| \geq N\left(|\hat{S}(n)| \leq \frac{1}{2}\right)\right\},
\end{array}
$$

where $P M(K)$ are the pseudomeasures (distributions with bounded Fourier coefficients) with support contained in $K$. Then the compact sets of multiplicity (三 not of uniqueness) are contained in $\bigcup_{N=1}^{\infty} F_{N}$, so it is enough to show that $\mathcal{K}(\mathbf{T})-\bigcup_{N=1}^{\infty} F_{N}$ is dense, since $F_{\mathcal{N}}$ is closed. But note that this set contains all finite sets of rationals so we are done.

ARGUMENT 2. We show that the class of compact $H$-sets (again see $[\mathbf{K}-\mathbf{S}]$ ) is comeager. Since these are all sets of uniqueness we are done. (One can prove by similar arguments here stronger facts, like for example that the class of Kronecker sets is comeager.)

Notice first that given pairwise disjoint intervals $I_{1} \cdots I_{k}$ and an interval $\Delta$ there are arbitrarily large enough $n$ and intervals $J_{1} \subseteq I_{1}, \ldots, J_{k} \subseteq I_{k}$ such that $n \cdot\left(\bigcup_{i=1}^{k} J_{i}\right) \subseteq J$. Intervals here are say in $(0,2 \pi)$ and multiplication $n \cdot x$ is modulo $2 \pi$.

Consider now the game in which players I, II take turns in playing at each move a finite sequence $I_{1} \cdots I_{k}$ of pairwise disjoint closed intervals with the only requirement that if $I$ played at some stage $I_{1} \cdots I_{k}$ then II must play next $J_{1}^{1} \cdots J_{n_{1}}^{1}, \ldots, J_{1}^{k}$ $\cdots J_{n_{k}}^{k}$ where $J_{m}^{i} \subseteq I_{i}$ and $n_{1}, \ldots, n_{k} \geq 1$. (Similarly for II.) If $K_{n}$ is the union of the intervals played in the $n$th move, let $K=\bigcap_{n} K_{n}$. II wins if $K$ is an $H$-set. It 
is easy to see that if II has a winning strategy in this game then the class of $H$-sets is comeager.

To show that II has a winning strategy fix some interval $\Delta$ in advance and let II play as follows: On the side II plays (secretly) in his $n$th move an integer $k_{n}$. If I played in his $n$th move $I_{1} \cdots I_{k}$ then II uses the observation before to play $J_{1} \cdots J_{k}, J_{i} \subseteq I_{i}$ and plays $k_{n}>k_{n-1}$ such that $k_{n} \cdot\left(J_{1} \cup \cdots \cup J_{k}\right) \subseteq \Delta$. If $K$ is the closed set produced at the end of the game we clearly have $k_{n} \cdot K \subseteq \Delta$ for all $n$, so $k_{0}<k_{1}<\cdots$ and any interval disjoint from $\Delta$ are witnesses that $K$ is an $H$-set.

We can also characterize completely the Borel $\sigma$-ideals with $\boldsymbol{\Sigma}_{2}^{0}$-basis.

THEOREM 13. Let I be a Borel (hence $\mathbf{\Pi}_{2}^{0}$ ) $\sigma$-ideal of compact sets in a compact metrizable space $E$. Then $I$ has a $\Sigma_{2}^{0}$-basis iff $I=\mathcal{K}(A)$, where $A$ is $\Delta_{2}^{0}$ in $E$.

Proof. If $A$ is $\boldsymbol{\Sigma}_{2}^{0}$ and $I=K(A)$ then $I$ clearly has a $\boldsymbol{\Sigma}_{2}^{0}$-basis.

If now $I$ has a $\Sigma_{2}^{0}$-basis, let $A_{I}=\{x:\{x\} \in I\} . A_{I}$ is $\boldsymbol{\Pi}_{2}^{0}$ as $I$ is, and $A_{I}$ is $\Sigma_{2}^{0}$ because if $B$ is some hereditary $\Sigma_{2}^{0}$-basis for $I, x \in A_{I} \Leftrightarrow\{x\} \in B$. So it remains to show that $I=\mathcal{K}\left(A_{I}\right)$. Let $U_{0}=\bigcup\left\{U\right.$ open in $E: U \cap A_{I}$ is a countable union of elements of $I\}$. Clearly $U_{0}$ is the largest open set in this family. We claim that $U_{0}=E$. Suppose not, and let $X=E-U_{0}, I_{X}=I \cap \mathcal{K}(X)$. If $K \in I_{X}, K$ must be rare in $X$, for if $V$ is open and $V \cap X \subseteq K$, i.e. $V \subseteq K \cup U_{0}$, then by maximality of $U_{0}, V \subseteq U_{0}$ hence $V \cap X=\varnothing$. So we can apply Lemma 11 and get that $I_{X}-\{\varnothing\}$ is meager in $\mathcal{K}(X)$. On the other hand, $A_{I} \cap X$ is dense in $X$, for if $V$ open satisfies $V \cap A_{I} \cap X=\varnothing$, i.e. $V \cap A_{I} \subseteq U_{0}$, then again by maximality $V \subseteq U_{0}$, i.e. $V \cap X=\varnothing$. But then the finite subsets of $A_{I} \cap X$, and a fortiori $I_{X}$, form a dense subset of $\mathcal{K}(X)$. As $I_{X}$ is $\Pi_{2}^{0}, I_{X}$ is comeager in $\mathcal{K}(X)$, a contradiction which shows $X=\varnothing$. So $U_{0}=E$ and $A_{I}$ is a countable union of elements of $I$, hence $I=\mathcal{K}\left(A_{I}\right)$.

As an immediate corollary we have

COROLLARY 14. If $E$ is a perfect compact metrizable space and $\gamma$ a subadditive capacity (in particular a measure) with $\gamma(\{x\})=0, \forall x \in E$, then the $\sigma$-ideal $I_{\gamma}$ of compact 0 sets of $\gamma$ does not have a $\boldsymbol{\Sigma}_{2}^{0}$-basis. Similarly the $\sigma$-ideal of nowhere dense closed sets on a perfect $E$ has no $\Sigma_{2}^{0}$-basis.

We have also

COROLLARY 15. Let $E$ be compact metrizable, and I a $\sigma$-ideal of compact sets on $E$, which is nontrivial, i.e. contains all singletons but not $E$. Then if $I$ has a $\boldsymbol{\Sigma}_{2}^{0}$-basis, I is complete $\boldsymbol{\Pi}_{1}^{1}$.

ProOF. If $I$ has a $\Sigma_{2}^{0}$-basis it has a hereditary $\Sigma_{2}^{0}$-basis so $I$ is $\Pi_{1}^{1}$. If it is not complete then it is $\Pi_{2}^{0}$ so by Theorem $13, I=\mathcal{K}\left(A_{I}\right)$ contradicting the nontriviality if $I$.

So this shows that the existence of a simple enough basis implies that the $\sigma$-ideal must be complicated. Finally note that if $I$ is a $\Pi_{1}^{1} \sigma$-ideal, $\left\{J_{n}\right\}$ and $D=E$ satisfy (a), (b) of Lemma 7 and $E \notin I$, then $I$ cannot have a $\Sigma_{2}^{0}$-basis. This is because either $I$ is true $\Pi_{1}^{1}$ and we can use Lemma 7 or else $I$ is Borel and we can use Theorem 13 (note that by (a), (b) $\{x\} \in I, \forall x \in E$ ). 


\section{Thinness of $\sigma$-ideals.}

3.1 Motivation and background. The notion of thinness has been introduced and extensively studied in the theory of capacities.

Recall that a function $\gamma: \mathcal{P}(E) \mapsto \mathbf{R}^{+}$is a capacity on the compact metrizable space $E$ if

(i) $\gamma(\varnothing)=0$ and $A \subseteq B \Rightarrow \gamma(A) \subseteq \gamma(B)$.

(ii) $\gamma\left(\bigcup_{n} A_{n}\right)=\sup _{n} \gamma\left(A_{n}\right)$, if $A_{0} \subseteq A_{1} \subseteq A_{2} \subseteq \cdots$.

(iii) $\gamma\left(\bigcap_{n} K_{n}\right)=\inf _{n} \gamma\left(K_{n}\right)$, if $K_{0} \supseteq K_{1} \supseteq K_{2} \supseteq \cdots$ are compact sets.

In the presence of (i) property (iii) is in fact equivalent to the restriction of $\gamma$ to $\mathcal{K}(E)$ being l.s.c., i.e. for $K \in \mathcal{K}(E), \gamma(K)<t \in \mathbf{R}_{+} \Rightarrow \exists$ open $U \supseteq K, \gamma(U)<t$. So

$$
I_{\gamma}=\{K \in \mathcal{K}(E): \gamma(K)=0\}
$$

is always a $\Pi_{2}^{0}$ set, and if $\gamma$ is subadditive i.e. $\gamma(A \cup B) \leq \gamma(A)+\gamma(B)$ then $I_{\gamma}$ is a $\sigma$-ideal.

The main result on capacities is Choquet's theorem [Ch]: If $A \subseteq E$ is $\boldsymbol{\Sigma}_{1}^{1}, \gamma(A)$ is the supremum of $\{\gamma(K): K \in \mathcal{K}(A)\}$. Note that this sup is taken over a $\boldsymbol{\Pi}_{2}^{1}$ set (sometimes a complete one). But in fact one can prove that if $A=\pi P$, where $P$ is $\Pi_{2}^{0}$ in $E \times 2^{\omega}$, the sup can be taken on $\left\{K: K=\pi K^{\prime}\right.$, for some $\left.K^{\prime} \in \mathcal{K}(P)\right\}$ which is a $\Sigma_{1}^{1}$ set. Therefore $\left\{A \in \Sigma_{1}^{1}: \gamma(A)=0\right\}$ is a $\Pi_{1}^{1}$ set (in fact the relation $\gamma(A)>t$ is $\Sigma_{1}^{1}$ in the codes of $\Sigma_{1}^{1}$ sets). Moreover by the outer capacitability theorem (Dellacherie [D1]), if $A$ is $\Sigma_{1}^{1}$ and $\gamma(A)=0$, there is Borel $B \supseteq A$ with $\gamma(B)=0$, i.e. $A$ is in the hereditary closure of

$$
\tilde{I}_{\gamma}=\{B \in \text { Borel }(E): \gamma(B)=0\} .
$$

Associated with the capacity $\gamma$ is a thickness function $e_{\gamma}$, defined by

$$
\begin{aligned}
e_{\gamma}(A)=\sup \{t & \in \mathbf{R}^{+}: \exists \Phi \text { uncountable } \\
& (\Phi \subseteq \mathcal{K}(A) \text { consists of pairwise } \\
& \text { disjoint sets } \& \gamma(K)>t, \forall K \in \Phi)\} .
\end{aligned}
$$

A set $A$ is $\gamma$-thin if $e_{\gamma}(A)=0$, i.e. there is no uncountable family of pairwise disjoint compact subsets of $A$ of positive capacity. If $E$ itself is $\gamma$-thin, we call $\gamma$ thin. When $\gamma$ is subadditive so that $\tilde{I}_{\gamma}$ is a $\sigma$-ideal, then we have that $\gamma$ is thin $\Leftrightarrow \operatorname{Borel}(E) / \tilde{I}_{\gamma}$ satisfies the countable chain condition.

The main result concerning thinness is due to Dellacherie [D1]: If $A \subseteq E$ is $\Sigma_{1}^{1}$, then

$$
e_{\gamma}(A)=\sup \left\{t \in \mathbf{R}^{+}: \exists \Phi \subseteq \mathcal{K}(A)\right.
$$

( $\Phi$ is perfect consisting of pairwise disjoint sets $\& \forall K \in \Phi(\gamma(K)>t)\}$.

It follows easily that

$$
J_{\gamma}=\{K \in \mathcal{K}(E): K \text { is } \gamma \text {-thin }\}
$$

is a $\Pi_{1}^{1} \sigma$-ideal, and using the same trick as above, that the relation $e_{\gamma}(A)>t$ is $\boldsymbol{\Sigma}_{1}^{1}$ on $\boldsymbol{\Sigma}_{1}^{1}$ sets in $E$.

From this it follows that for a capacity $\gamma$, the following are equivalent:

(i) $\gamma$ is thin.

(ii) If $H \subseteq E$ is $\Pi_{2}^{0}$ then there is $F \subseteq H, F$ in $\Sigma_{2}^{0}$ with $\gamma(H-F)=0$ (Feyel). 
(iii) If $A \subseteq E$ is $\Sigma_{1}^{1}$ there is $B$ Borel, $B \subseteq A$ with $\gamma(A-B)=0$ (Dellacherie).

(iv) For some fixed $\xi$, every Borel set $B$ contains a Borel set $B^{\prime} \subseteq B$ of rank $\leq \xi$ with $\gamma\left(B-B^{\prime}\right)=0$ (Louveau) (see $[\mathbf{D F M}]$ ).

The previous results can be extended to the following situations:

Give the space

$$
E^{+}=\left\{\mu \in \mathcal{M}^{+}(E): \mu(E) \leq 1\right\}
$$

the weak*-topology, for which it is compact metrizable. For $H \subseteq E^{+}$let $\gamma_{H}: \mathcal{P}(E)$ $\rightarrow[0,1]$ be defined by

$$
\gamma_{H}(A)=\sup _{\mu \in H} \mu^{*}(A)
$$

If $H$ is compact, $\gamma_{H}$ is a subadditive capacity (but the converse is not true). If now $H$ is $\Sigma_{1}^{1}$ in $E^{+}$we call $\gamma_{H}$ an analytic submeasure. In [D3] Dellacherie extends all the results quoted above to such analytic submeasures, except that in this case the $\sigma$-ideal $I_{\gamma_{H}}$ may be complete $\Pi_{1}^{1}$. An interesting example of such a situation occurs in the theory of sets of uniqueness. Taking $E=\mathbf{T}=$ the unit circle, let $H=R^{+}=$ the positive Rajchman measures $=\left\{\mu \in E^{+}: \hat{\mu}(n) \rightarrow 0\right\}$. Then $I_{\gamma_{R^{+}}}=U_{0} \equiv$ the compact extended uniqueness sets. Solovay $[\mathbf{S}]$ and independently Kaufman $[\mathbf{K 1}]$ have shown that $U_{0}$ is complete $\Pi_{1}^{1}$.

Moreover in this context one has the following.

For an analytic submeasure $\gamma=\gamma_{H}$ on $E$ the following are equivalent:

(i) $\gamma$ is thin. [D2]).

(ii) There is a measure $\mu$ which controls $\gamma$, i.e. $\mu(K)=0 \Rightarrow \gamma(K)=0$ (Dellacherie

(iii) $I_{\gamma}=I_{\mu}$, for some measure $\mu$ (from a result of Mokobodzki, see [DFM]).

(iv) $\tilde{I}_{\gamma}=\{B \in$ Borel $(E): \gamma(B)=0\}$ is Borel (in the codes of Borel sets) (Louveau [L2]).

Notice that from (i), (ii) it follows that if a measure controls $\gamma_{H}$ then $I_{\gamma_{H}}$ is Borel (being equal to $I_{\mu}$, for some $\mu$ ). In particular no measure can control $\gamma_{R^{+}}$ i.e. if $\mu$ is any measure on $\mathbf{T}$ then there is a compact $\mu$-measure 0 set which is not in $U_{0}$ i.e. is of restricted multiplicity. (This is a known fact-we are only making the point here that it is also a consequence of the classification of $U_{0}$ as true $\boldsymbol{\Pi}_{1}^{1}$.)

In the following subsections we will give abstract versions of almost all these results, in the context of $\boldsymbol{\Pi}_{1}^{1} \sigma$-ideals.

3.2 Extending $\sigma$-ideals of compact sets. Let $I$ be a $\sigma$-ideal of compact sets on a compact metrizable space $E$. We say that a $\sigma$-ideal of sets $I$ extends $I$ if $I \cap K(E)$ $=I$.

Of course there is a smallest $\sigma$-ideal extending $I$, namely $I_{\sigma}=\left\{A \in \mathcal{P}(E): \exists\left\{K_{n}\right\}\right.$ $\left.\left(\forall n\left(K_{n} \in I\right) \& A \subseteq \bigcup_{n} K_{n}\right)\right\}$ (there is an abuse of notation here since for each $X$ we denote usually by $X_{\sigma}$ the set $\left\{A: \exists\left\{A_{n}\right\}\left[\forall n\left(A_{n} \in X\right) \& A=\bigcup_{n} A_{n}\right]\right\}$ but this will cause no confusion here). The restriction of $I_{\sigma}$ on $\Sigma_{2}^{0}(E)$ is the unique extension of $I$ to $\Sigma_{2}^{0}(E)$, i.e. any $\sigma$-ideal $I$ extending $I$ must satisfy $I \cap \Sigma_{2}^{0}(E)=I_{\sigma} \cap \boldsymbol{\Sigma}_{2}^{0}(E)$. Note also that if $I$ is $\Pi_{2}^{0}$, resp. $\Pi_{1}^{1}$ in $\mathcal{K}(E), I_{\sigma} \cap \mathcal{K}_{\sigma}(E)$ is $\boldsymbol{\Pi}_{2}^{0}$, resp. $\Pi_{1}^{1}$ in the codes of $\Sigma_{2}^{0}$ sets.

No such uniqueness holds in general for $\Pi_{2}^{0}(E)$. We say however that a $\sigma$-ideal I of $\Pi_{2}^{0}$ sets (resp. of Borel sets, etc.) has the inner approximation property if for every $\mathbf{\Pi}_{2}^{0}$ (resp. Borel, etc.) set $H \subseteq E$,

$$
H \notin I \Rightarrow \exists K \in \mathcal{K}(E)(K \subseteq H \& K \notin I) .
$$


For example the $\sigma$-ideals $\tilde{I}_{\gamma}$ for $\gamma$ a subadditive capacity or a submeasure have this property. We now have the following

Proposition 1. Let $I$ be a $\sigma$-ideal of compact sets in a compact metrizable space $E$. Then the following are equivalent.

(i) There is a $\sigma$-ideal I of $\Pi_{2}^{0}$ sets extending $I$ and having the inner approximation property.

(ii) $\tilde{I}_{\mathbf{\Pi}_{2}^{0}}=\left\{H \in \mathbf{\Pi}_{2}^{0}(E): \forall K \in \mathcal{K}(H)(K \in I)\right\}$ is a $\sigma$-ideal.

(iii) If $F \in I_{\sigma} \cap \Sigma_{2}^{0}(E), H \in \tilde{I}_{\Pi_{2}^{0}}$ and $K=F \cup H \in \mathcal{K}(E)$, then $K \in I$.

In this case, $\tilde{I}_{\Pi_{2}^{0}}$ is the unique $\sigma$-ideal of $\Pi_{2}^{0}$ sets extending $I$ and having the inner approximation property.

DEFINITION. We say that a $\sigma$-ideal $I$ of compact sets in a compact metrizable space is calibrated if it satisfies (any of) the conditions of Proposition 1.

Thus the $\sigma$-ideals $I_{\gamma}$ for $\gamma$ a subadditive capacity or a submeasure are calibrated. We conjecture that the $\sigma$-ideal of closed sets of uniqueness is also calibrated. On the other hand the $\sigma$-ideal of nowhere dense closed sets of $2^{\omega}$ is not calibrated.

We prove now Proposition 1.

Proof of Proposition 1. Clearly (ii) $\Rightarrow$ (i). To see that (i) $\Rightarrow$ (ii) note that if $I \subseteq \Pi_{2}^{0}(E)$ extends $I$, then $I \subseteq \tilde{I}_{\boldsymbol{\Pi}_{2}^{0}}$ and if $I$ has the inner approximation property $\tilde{I}_{\boldsymbol{\Pi}_{2}^{0}} \supseteq I$. Moreover (ii) $\Rightarrow$ (iii) is obvious. So it remains only to prove (iii) $\Rightarrow$ (ii).

Assume (iii) and let $\left\{H_{n}\right\}$ be a sequence of $\Pi_{2}^{0}$ sets in $\tilde{I}_{\Pi_{2}^{0}}$. Let $H \subseteq \bigcup_{n} H_{n}, H_{n}$ $\in \tilde{I}_{\mathbf{\Pi}_{2}^{0}}$. We want to prove $H \in \tilde{I}_{\mathbf{\Pi}_{2}^{0}}$, and for that it is enough to prove that if $K \in \mathcal{K}(E), K \subseteq \bigcup_{n} H_{n}$ then $K \in I$. Assume not, towards a contradiction. Let $E-H_{n}=\bigcup_{p} K_{n}^{p}$, with $K_{n}^{p} \in \mathcal{K}(E)$. Then $K=\left(K \cap H_{0}\right) \cup \bigcup_{p}\left(K \cap K_{0}^{p}\right)$ and $K \cap H_{0} \in \tilde{I}_{\mathbf{\Pi}_{2}^{\mathbf{0}}}$, so by (iii) there is $p_{0}$ with $K \cap K_{0}^{p_{0}} \notin I$. Now $K \cap K_{0}^{p_{0}}=\left(K \cap K_{0}^{p_{0}} \cap\right.$ $\left.H_{1}\right) \cup \bigcup_{p}\left(K \cap K_{0}^{p_{0}} \cap K_{1}^{p}\right)$, so again there is $p_{1}$ with $K \cap K_{0}^{p_{0}} \cap K_{1}^{p_{1}} \notin I$, etc. So we can construct a sequence $\left\{p_{i}\right\}$ with $K \cap \bigcap_{i<n} K_{i}^{p_{i}} \notin I$, all $n$. Thus $K \cap \bigcap_{i<n} K_{i}^{p_{i}} \neq \varnothing$, all $n$ and so $K \cap \bigcap_{i \in \omega} K_{i}^{p_{i}} \neq \varnothing$. This contradicts $K \subseteq \bigcup_{n} H_{n}$.

3.3 Thinness and approximation properties. Let $I$ be a $\sigma$-ideal of compact sets on a compact metrizable space $E$. A set $A \subseteq E$ is $I$-thin if there is no uncountable family $\Phi \subseteq \mathcal{K}(A)$ of pairwise disjoint sets which are not in $I$. In case of $I=I_{\gamma}$ as 3.1 this corresponds to the usual concept of thinness. We say that $I$ is thin if $E$ is I-thin.

One can prove for this abstract notion an analog of the result for thickness functions.

THEOREM 2. Let I be a $\Pi_{1}^{1} \sigma$-ideal of compact sets in a compact metrizable space $E$. Let $A \subseteq E$ be $\Pi_{2}^{0}$. If $A$ is not $I$-thin there is a continuous function $\varphi: 2^{\omega} \rightarrow \mathcal{K}(A)$ such that (i) $\forall \alpha \in 2^{\omega}(\varphi(\alpha) \notin I) \&$ (ii) $\forall \alpha, \beta \in 2^{\omega}(\alpha \neq \beta \Rightarrow$ $\varphi(\alpha) \cap \varphi(\beta)=\varnothing)$.

Such a $\varphi$ will be called a thickness witness for $A$.

The proof of this theorem follows easily from the following lemma of Mokobodzki (unpublished, see [DFM]), independently rediscovered and used for other purposes by many authors (Burgess-Mauldin [BM], Louveau [L2]). 
LEMMA 3. Let $P$ be a Polish space, $R$ a $\Sigma_{2}^{0}$ symmetric reflective relation on $P$. Let $A \subseteq P$ be $\Sigma_{1}^{1}$. If there is an uncountable subset of $A$ consisting of pairwise not in $R$ elements, there exists such a perfect set.

Proof. Let first $Q$ be Polish and $\varphi: Q \rightarrow A$ a continuous surjection. Define $R^{\prime}$ on $Q$ by $(x, y) \in R^{\prime} \Leftrightarrow(\varphi(x), \varphi(y)) \in R$. Then $Q, R^{\prime}, A^{\prime}=Q$ satisfy the hypotheses of the lemma, and if $F \subseteq Q$ is perfect and consists of pairwise not in $R^{\prime}$ elements, so does $\varphi^{\prime \prime}(F)$ relative to $P, R$. So we may assume $A=P$. If there is an uncountable subset of $P$ of pairwise not in $R$ elements, there is an uncountable dense in itself such set, say $D \subseteq P$. Let $R=\bigcup_{n} R_{n}$ where each $R_{n}$ is symmetric, reflexive and closed in $P^{2}$ and $R_{0} \subseteq R_{1} \subseteq R_{2} \subseteq \cdots$. Now perform the usual construction à la Cantor, using balls centered at points in $D$, of decreasing diameter, and insuring at the $n$th step that for pairs $(x, y)$ where $x, y$ are in different balls, $(x, y) \notin R_{n}$. This gives the desired perfect set.

ProOF OF THEOREM 2. Apply Lemma 3 to $\mathcal{K}(A),\{K \in \mathcal{K}(A): K \notin I\}$, and the closed relation $R=\left\{\left(K, K^{\prime}\right) \in \mathcal{K}(A)^{2}: K \cap K^{\prime} \neq \varnothing\right\} \cup\{(\varnothing, \varnothing)\}$.

Note that we never used the fact that $I$ is a $\sigma$-ideal.

COROLLARY 4. Let I be a $\Pi_{1}^{1} \sigma$-ideal of compact sets in a compact metrizable space $E$. Then

(i) If $J_{I}=\{K \in K(E): K$ is $I$-thin $\}$, then $J_{I}$ is a $\Pi_{1}^{1} \sigma$-ideal.

(ii) If $I$ is calibrated, so is $J_{I}$.

Proof. (i) By Theorem $2 K \notin J_{I} \Leftrightarrow K$ has a thickness witness, and this is $\Sigma_{1}^{1}$. If $K_{n} \in J_{I}$ for all $n$, and $K=\bigcup_{n} K_{n}$ is not $I$-thin, then let $\left\{K_{\alpha}\right\}_{\alpha \in 2^{\omega}}$ be a thickness witness for $K$. Let $D_{n}=\left\{\alpha \in 2^{\omega}: K_{n} \cap K_{\alpha} \notin I\right\}$. Then $D_{n}$ is countable, so let $\alpha_{0} \notin \bigcup_{n} D_{n}$, i.e. $K_{\alpha_{0}} \cap K_{n} \in I$ for all $n$, therefore $K_{\alpha_{0}} \in I$, a contradiction.

(ii) Suppose $H \in \widetilde{\left(J_{I}\right)_{\Pi_{2}^{0}}}, K_{n} \in J_{I}$ and $K=H \cup K_{n}$ is not $I$-thin. Let $\left\{K_{\alpha}\right\}_{\alpha \in 2^{\omega}}$ be a thickness witness for $K$. Again $D=\bigcup_{n} D_{n}=\left\{\alpha: \exists n\left(K_{n} \cap K_{\alpha} \notin I\right)\right\}$ is countable. If $\alpha \notin D$, then $K_{\alpha}=\left(K_{\alpha} \cap H\right) \cup \bigcup_{n}\left(K_{n} \cap K_{\alpha}\right)$ is not in $I$ but for all $n K_{\alpha} \cap K_{n} \in I$. Since $I$ is calibrated there is $K_{\alpha}^{\prime} \subseteq K_{\alpha} \cap H$ with $K_{\alpha}^{\prime} \notin I$. So $H$ is not $I$-thin contradicting Theorem 2, which implies that then $H$ must contain a closed non- $I$-thin set.

A stronger result can be proved if the ideal $I$ has a stronger calibration property.

DEFINITION. Let $I$ be a $\sigma$-ideal of compact sets in a compact metrizable space $E$. We say that $I$ is strongly calibrated if for every $K \notin I$ and any $P \in \Pi_{2}^{0}$, $P \subseteq E \times 2^{\omega}$ with $\pi P=K$ there is $K^{\prime} \in K(P)$ with $\pi K^{\prime} \notin I$. (Notice that it is equivalent to have $P \in \Sigma_{1}^{1}$.)

This concept essentially comes from [D1]. Note first that strongly calibrated $\Rightarrow$ calibrated. (If $K=H \cup \bigcup_{n} K_{n}, K_{n} \in I, H \in \tilde{I}_{\Pi_{2}^{0}}$, let $P=H \times\{0\} \cup \bigcup_{n}\left(K_{n} \times\{n\}\right)$. If $K \notin I$ let $K^{\prime}$ be as in the definition of "strongly calibrated" and let $K^{*}=$ $\pi\left(K^{\prime} \cap(E \times\{\varnothing\})\right.$.) Then $\pi K^{\prime} \subseteq \bigcup_{n} K_{n} \cup K^{*}$ so $K^{*} \notin I$. But $K^{*} \subseteq H$, a contradiction.) Note also that $I_{\gamma}$, for a capacity or submeasure $\gamma$, is strongly calibrated.

We have now the following

COROLLARY 5. Let I be a strongly calibrated $\sigma$-ideal of compact sets in a compact metrizable space $E$. Then

(i) $J_{I}$ is strongly calibrated. 
(ii) If $A \in \Sigma_{1}^{1}$ is not $I$-thin then $A$ contains a thickness witness and

$$
I_{I}=\left\{A \in \Sigma_{1}^{1}(E): A \text { is } I \text {-thin }\right\}
$$

is a $\sigma$-ideal of $\Sigma_{1}^{1}$ sets extending $J_{I}$.

Proof. (i) Let $K \notin J_{I}, P \subseteq E \times 2^{\omega}, \pi P=K$. Let $\left\{K_{\alpha}\right\}_{\alpha \in 2^{\omega}}$ be a thickness witness for $P$. Then if $K_{\alpha}^{\prime}=\left(K_{\alpha} \times 2^{\omega}\right) \cap P, \pi K_{\alpha}^{\prime}=K_{\alpha^{\prime}}$, so choose $K_{\alpha}^{*} \subseteq K_{\alpha}^{\prime}$ with $\pi K_{\alpha}^{*} \notin I$. As in the proof of Theorem 2 it follows that there is $\varphi: 2^{\omega} \rightarrow \mathcal{K}(P)$ with $\pi \varphi(\alpha) \cap \pi \varphi(B)=\varnothing$ if $\alpha \neq \beta$ and $\pi \varphi(\alpha) \notin I$. This completes clearly the proof.

(ii) If $A \in \Sigma_{1}^{1}, P \subseteq E \times 2^{\omega}$ is $\Pi_{2}^{0}$ with $\pi P=A$ then we have as before

$A \in I_{I} \Leftrightarrow \exists F \in \mathcal{K}(P) \exists L[L \in \mathcal{K}(K(F)) \& L$ is perfect

$$
\left.\& \forall K \forall K^{\prime}\left(K \in L \& K^{\prime} \in L \& K \neq K^{\prime} \Rightarrow \pi K \cap \pi K^{\prime}=\varnothing\right) \& \pi(\bigcup L) \notin I\right]
$$

which is clearly $\Sigma_{1}^{1}$.

Suppose now $\left\{A_{n}\right\}$ are $I$-thin $\Sigma_{1}^{1}$ sets and let $\left\{P_{n}\right\}$ be $\Pi_{2}^{0}$ sets in $E \times 2^{\omega}$ with $\pi P_{n}=A_{n}$. Let $P=\bigcup_{n}\left(P_{n} \times\{n\}\right)$ in $E \times 2^{\omega} \times(\omega+1)$ (viewed as a subset of $\left.E \times 2^{\omega}\right)$. Then $\pi P=\bigcup_{n} A_{n}=A$. So if $A$ is not $I$-thin, we have as before a thickness witness $L \subseteq \mathcal{K}(P)$. For each $K \in L$ let $K_{n}=K \cap\left(E \times 2^{\omega} \times\{n\}\right)$. For each $n$, all but countably many of the $\pi K_{n}$ must be in $I$, since $A_{n}$ is $I$-thin. But this easily contradicts that for $K \in L, \pi K \notin I$. So $A$ is $I$-thin.

It is an interesting question to find out for a given $I$ whether $I=J_{I}$ i.e. whether every compact $I$-thin set is actually in $I$. This does not happen for example if $I$ is thin (and does not contain all compact sets), e.g. if $I=I_{H}$ for a controlled $\Sigma_{1}^{1}$-submeasure $H$. For capacities $\gamma$ we have mentioned in $\S 2.3$ that $J_{\gamma_{0}}=I_{\gamma_{0}}$ if $\gamma_{0}$ is the electrostatic capacity, but $J_{\gamma} \neq I_{\gamma}$ for the capacity in Proposition 2.9. For nonthin submeasures an interesting case is $H=R^{+}=$the positive Rajchman measures. Kaufman [K2] has shown that $J_{\gamma_{R^{+}}}=I_{\gamma_{R^{+}}}=U_{0}$. It follows that the $\sigma$-ideal $I_{\gamma_{R^{+}}}$of $\Sigma_{1}^{1} I_{\gamma_{R^{+}}}$-thin sets is exactly the $\sigma$-ideal of $\Sigma_{1}^{1}$ zero sets for $\gamma_{R^{+}}$, i.e. the class of $\Sigma_{1}^{1}$ extended uniqueness sets. The case $I=U=$ the $\sigma$-ideal of compact uniqueness sets is also of particular interest. Kaufman [K2] has also shown that $J_{U}=U$ in this case as well.

We discuss now approximation properties. Recall that for each $\sigma$-ideal of sets $I$ on $E$ we denote $\tilde{I}=\{A \subseteq E: \mathcal{K}(A) \subseteq I\}, \tilde{I}_{\Pi_{2}^{0}}=\tilde{I} \cap \Pi_{2}^{0}, \tilde{I}_{\text {Borel }}=\tilde{I} \cap$ Borel, etc.

Proposition 6. Let I be a thin $\sigma$-ideal of compact sets in a compact metrizable space $E$. Then

(i) If $H \subseteq E$ is $\boldsymbol{\Pi}_{2}^{0}$, there is $\boldsymbol{\Sigma}_{2}^{0} F \subseteq H$ with $H-F \in \tilde{I}_{\Pi_{2}^{0}}$.

(ii) If $\tilde{I}_{\text {Borel }}$ is a $\sigma$-ideal, then for any $A \subseteq E$ in $\Pi_{1}^{1}$ there exist Borel sets $B_{1}, B_{2}$ such that $B_{1} \subseteq A \subseteq B_{2}$ and $\left(B_{2}-B_{1}\right) \in \tilde{I}_{\text {Borel }}$.

ProOF. (i) Let $\left\{K_{n}\right\}$ be a maximal family of pairwise disjoint compact subsets of $H$ which are not in $I$ (since $I$ is thin this is countable). Let $F=\bigcup_{n} K_{n}$. Then by maximality, $H-F \in \tilde{I}_{\Pi_{2}^{0}}$. (Note that we only used the thinness of $H$ here.)

(ii) Let $A$ be $\Pi_{1}^{1}$ in $E$, and let $x \mapsto T_{x} \subseteq \omega^{\omega}$ be a Borel function associating to each $x \in E$ a tree $T_{x}$ on $\omega$ such that $x \in A \Leftrightarrow T_{x}$ is well-founded. Let $A_{\xi}=$ $\left\{x:\left|T_{x}\right|<\xi\right\}$, and $A_{\leq \xi}=\left\{x:\left|T_{x}\right| \leq \xi\right\}$, where $\xi<\omega_{1}$ and $|T|$ is the rank of the well-founded tree $T$. Since the $A_{\xi}$ 's are pairwise disjoint, and $I$ is thin, we 
must have $A_{\xi} \in \tilde{I}_{\text {Borel }}$, for all $\xi \geq \xi_{0}$ (some $\xi_{0}<\omega_{1}$ ). Similarly for $u \in \omega^{<\omega}$ let $A_{\xi}^{u}=\left\{x:\left|T_{x}^{u}\right|=\xi\right\}$ where $T_{x}^{u}=\left\{v: u \widehat{v} \in T_{x}\right\}$. Again $A_{\xi}^{u} \in \tilde{I}_{\text {Borel }}$ for $\xi \geq \xi_{0}^{u}$. Let $\xi_{1}=\sup _{u} \xi_{0}^{u}<\omega_{1}$. Then let $B_{1}=A_{\leq \xi_{1}}$ and $B_{2}=B_{1} \cup \bigcup_{u} A_{\xi_{1}}^{u}$. Then $B_{1} \subseteq A \subseteq B_{2}$, since if $x \in A-B_{1}$, then $\left|T_{x}\right|>\xi_{1}$ so for some $u \in T_{x},\left|T_{x}^{u}\right|=\xi_{1}$. Since $\tilde{I}_{\text {Borel }}$ is a $\sigma$-ideal, $B_{2}-B_{1} \subseteq \bigcup_{u} A_{\xi}^{u}$ is in $\tilde{I}_{\text {Borel }}$.

Proposition 5 admits a kind of converse, which generalizes the Dellacherie-Feyel results on capacities.

THEOREM 7. (i) Let I be a calibrated $\mathbf{\Pi}_{2}^{0} \sigma$-ideal of compact sets in a compact metrizable space $E$. If

$$
\text { For each } \Pi_{2}^{0} H \subseteq E \text { there is } \boldsymbol{\Sigma}_{2}^{0} F \subseteq H \text { with } H-F \in \tilde{I}_{\Pi_{2}^{0}},
$$

then I is thin.

(ii) Suppose $I$ is a $\sigma$-ideal of compact sets in $E$ such that $\tilde{I}_{\text {Borel }}$ is a $\sigma$-ideal and is $\mathbf{\Pi}_{1}^{1}$ in the codes of Borel sets. If

$$
\begin{aligned}
& \text { For each } \Sigma_{1}^{1} \text { set } A \subseteq E \text { there exist Borel sets } B_{1}, B_{2} \text { with } B_{1} \subseteq \\
& A \subseteq B_{2} \text { and } B_{2}-B_{1} \in \tilde{I}_{\text {Borel }} \text {, }
\end{aligned}
$$

then $I$ is thin.

ProOF. (i) If $I$ is not thin let $\varphi: 2^{\omega} \rightarrow \mathcal{K}(E)$ be a thickness witness for $E$. If $H \subseteq 2^{\omega}$ is $\Pi_{2}^{0}$, let $H^{\prime}=\bigcup_{\alpha \in H} \varphi(\alpha)$. Then $H^{\prime}$ is $\Pi_{2}^{0}$ in $E$, so by $(*)$ there is

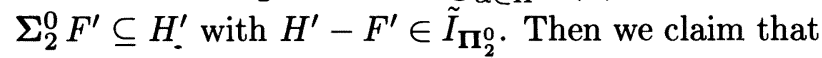

$$
\alpha \in H \Leftrightarrow F^{\prime} \cap \varphi(\alpha) \notin I_{\sigma} \cap \Sigma_{2}^{0}(E) .
$$

Granting this, we have that it is $\Sigma_{2}^{0}$, since $I$ is $\Pi_{2}^{0}$ and thus the relation $F^{\prime} \cap \varphi(\alpha) \in$ $I_{\sigma}$ is also $\Pi_{2}^{0}$. But $H$ was arbitrary $\Pi_{2}^{0}$, and we have a contradiction.

To prove the claim note that if $\alpha \in H$, then $\varphi(\alpha) \subseteq F^{\prime} \cup\left(H^{\prime}-F^{\prime}\right)$, so since $I$ is calibrated and $H^{\prime}-F^{\prime} \in \tilde{I}_{\Pi_{2}^{0}}$ we have that $F^{\prime} \cap \varphi(\alpha) \notin I_{\sigma}$. If $\alpha \notin H$ now, then $\varphi(\alpha) \cap F^{\prime} \subseteq \varphi(\alpha) \cap H^{\prime}=\varnothing$, so $\varphi(\alpha) \cap F^{\prime} \in I_{\sigma}$.

(ii) The proof is similar. Using again a thickness witness for $E$, and taking a $\boldsymbol{\Sigma}_{1}^{1}$ set $A \subseteq 2^{\omega}$ we get that $A^{\prime}=\bigcup_{\alpha \in A} \varphi(\alpha)$ is $\Sigma_{1}^{1}$, hence by $(* *)$ there are Borel $B_{1}^{\prime}$, $B_{2}^{\prime}$ with $B_{1}^{\prime} \subseteq A^{\prime} \subseteq B_{2}^{\prime}$ and $B_{2}^{\prime}-B_{1}^{\prime} \in \tilde{I}_{\text {Borel }}$. Using that $\tilde{I}_{\text {Borel }}$ is a $\sigma$-ideal, we get that

$$
\alpha \in A \Leftrightarrow \varphi(\alpha)-B_{2} \in \tilde{I}_{\text {Borel }},
$$

which by hypothesis is $\Pi_{1}^{1}$, and leads to a contradiction.

3.4 Controlling $\sigma$-ideals. Let $I$ be a $\sigma$-ideal of compact sets on $E$, and $A$ a collection of $\Pi_{2}^{0}$ subsets on $E$. We say that $A$ is compatible with $I$ if the least $\sigma$-ideal of $\Pi_{2}^{0}$ sets $I$ containing $I$ and $A$ extends $I$, i.e. satisfies $I \cap \mathcal{K}(E)=I$.

LEMMA 8. A set $A \subseteq \Pi_{2}^{0}(E)$ is compatible with $I$ iff for each $H \in A, F \in$ $I_{\sigma} \cap \Sigma_{2}^{0}(E), K \in \mathcal{K}(E)$,

$$
K \subseteq H \cup F \Rightarrow K \in I .
$$

Proof. The condition is clearly necessary, as such a $K$ must be in $I$. Conversely, if the condition is fulfilled, a capacitability argument as in Proposition 1 gives the result. 
For example, $I$ is calibrated iff $\tilde{I}_{\Pi_{2}^{0}}$ is compatible with $I$. Similarly condition (b) in Lemma 2.7 can be read: $\left(\widetilde{\cap J_{n}}\right)_{\Pi_{2}^{0}}$ is compatible with $I$. In case of a $\Sigma_{1}^{1}$ submeasure $\gamma$ and a measure $\mu$, the $\sigma$-ideal $I_{\mu}=\left\{H \in \Pi_{2}^{0}(E): \mu(H)=0\right\}$ is compatible with $I_{\gamma}=\{K: \gamma(K)=0\}$ iff $\mu$ controls the submeasure $\gamma$. More generally if $I, I^{\prime}$ are calibrated $\sigma$-ideals of compact sets then $I \subseteq I^{\prime} \Leftrightarrow \tilde{I}_{\Pi_{2}^{0}}$ is compatible with $I^{\prime}$.

DEFINITION. A $\sigma$-ideal of compact sets $I$ on a compact metrizable space $E$ is said to be controlled if there is $A \subseteq \Pi_{2}^{0}(E)$ such that $\varnothing \in A, A$ is compatible with $I$ and $A$ is $\Sigma_{1}^{1}$ in the codes of $\Pi_{2}^{0}$ sets. Such an $A$ is called a control set for $I$.

For example, if $\mu$ is a control measure for the $\Sigma_{1}^{1}$ submeasure $\gamma$, then $I_{\mu}$ is a control set for $I_{\gamma}$. Also, if the $\boldsymbol{\Sigma}_{1}^{1}$ submeasure $\gamma$ satisfies $A=\left\{H \in \boldsymbol{\Pi}_{2}^{0}(E): \gamma(H)=\right.$ $0\}$ is Borel in the codes, then $A$ controls $\gamma$. On the other hand, for $E$ uncountable, $\{\varnothing\}$ is not a control set since the relation $H=\varnothing$ is a true $\Pi_{1}^{1}$ relation in the codes of $\boldsymbol{\Pi}_{2}^{0}$ sets.

The next results can be thought of as abstract definability versions of the standard facts about controlled (by measures) submeasures.

THEOREM 9. Let I be a controlled $\Pi_{1}^{1} \sigma$-ideal of compact sets in a compact metrizable space. Then $I$ is thin.

This extends half of Dellacherie's result on control by measures.

THEOREM 10. Let $I$ be a controlled $\Pi_{1}^{1} \sigma$-ideal of compact sets in a compact metrizable space. Then $I$ is $\mathbf{\Pi}_{2}^{0}$.

Here is an immediate corollary.

COROLlaRY 11 (UPWARD PRESERVATION OF BoRELneSS). Let $I, I^{\prime}$ be two calibrated $\Pi_{1}^{1} \sigma$-ideals of compact sets in a compact metrizable space, with $I \subseteq I^{\prime}$. Then

$$
\tilde{I}_{\Pi_{2}^{0}} \text { is Borel in the codes } \Rightarrow I^{\prime} \text { is Borel }\left(\therefore \Pi_{2}^{0}\right) \text {. }
$$

In particular if $\mu$ is a measure and $I_{\mu} \subseteq I^{\prime}$ then $I^{\prime}$ is $\Pi_{2}^{0}$, i.e. any calibrated $\Pi_{1}^{1} \sigma$-ideal containing the 0 -sets of some measure is $\Pi_{2}^{0}$. This can be rephrased as follows.

If $I$ is a calibrated true $\Pi_{1}^{1} \sigma$-ideal and $\mu$ is any measure, then there is a compact set of $\mu$-measure 0 which is not in $I$.

So this can be viewed as an abstract definability version of the standard result in the theory of sets of uniqueness which says that for any measure $\mu$ there is a perfect set of $\mu$-measure 0 which is a set of restricted multiplicity (the case $\mu=$ Lebesgue measure is of course the famous construction of Menshov).

We prove now these two theorems.

ProOF OF THEOREM 9. Assume $E$ is not $I$-thin, towards a contradiction. Let $\varphi$ be a thickness witness for $E$. If $A$ is a control set for $I$, we have for each $\Pi_{2}^{0}$ subset $H$ of $2^{\omega}$,

$$
H=\varnothing \Leftrightarrow \bigcup_{\alpha \in H} \varphi(\alpha) \in A
$$

But the relation on the left side is complete $\Pi_{1}^{1}$, while the one on the right is $\Sigma_{1}^{1}$ by hypothesis, a contradiction. 
Note that in this proof we only needed that $A$ has the property: $K \subseteq G \in A \Rightarrow$ $K \in I$, for all $K \in \mathcal{K}(E)$, i.e. $A \subseteq \tilde{I}_{\Pi_{2}^{0}}$.

PROOF OF THEOREM 10 . We will need first a lemma on increasing families of $\Pi_{2}^{0}$ sets, which is an unpublished result of Choquet, and that we independently rediscovered.

Lemma 12 (Choquet). Let $A$ be a $\Pi_{1}^{1}$ set in a Polish space $P,\left\{A_{\xi}\right\}_{\xi<\omega_{1}}$ an increasing family of $\Pi_{2}^{0}$ sets with $A=\bigcup_{\xi} A_{\xi}$. Then $A$ is $\Pi_{2}^{0}$ in $P$.

REMARK. By Hausdorff's well-known construction $\left\{A_{\xi}\right\}$ need not be eventually constant.

ProOF. One can use Hurewicz's Theorem: If $A$ is not $\Pi_{2}^{0}, A$ must contain a copy $D$ of $\mathbf{Q}$ as a relatively closed subset. But $D$ is countable, so for some $\xi<\omega_{1}, D=D \cap A_{\xi}=D \cap A$ is relatively closed in $A_{\xi}$. Since $A_{\xi}$ (being $\Pi_{2}^{0}$ ) is Polish this is impossible. (This quick proof was noticed by Saint Raymond.)

One can also give a direct proof: Let $B=P-A$, and let $Q$ be Polish, $\varphi: Q \rightarrow B$ a continuous surjection. Let $U \subseteq Q$ be the largest open subset of $Q$ such that $\varphi^{\prime \prime}(U)$ is contained in a $\Sigma_{2}^{0}$ set disjoint from $A$. We want to show $U=Q$ (and so $\varphi^{\prime \prime}(U)=B$ ). So, by contradiction, let $Q^{\prime}=Q-U \neq \varnothing$, and let $\left\{V_{n}\right\}$ be a basis for nonempty open sets in $Q^{\prime}$. By an easy Baire category argument, there exists for each $\xi$ an integer $n$ with $\overline{\varphi\left(V_{n}\right)} \cap A_{\xi}=\varnothing$. Let $n_{\xi}$ be the least such. There must be $n_{0}$ such that $n_{\xi}=n_{0}$ for cofinally many $\xi$ 's and since the $A_{\xi}$ 's are increasing $\overline{\phi\left(V_{n_{0}}\right)} \cap \bigcup_{\xi} A_{\xi}=\varnothing$, hence $V_{n_{0}} \cup U$ contradicts the maximality of $U$.

We continue the proof of Theorem 10 now: Let $A$ control $I$, and let $B \subseteq I$ be Borel. Consider

$$
B^{\prime}=\left\{K \in \mathcal{K}(E): \exists\left\{K_{n}\right\}\left[\forall n\left(K_{n} \in B\right) \&\left(K-\bigcup_{n} K_{n}\right) \in A\right]\right\} .
$$

As $A$ is $\boldsymbol{\Sigma}_{1}^{1}$ in the codes, $B^{\prime}$ is $\boldsymbol{\Sigma}_{1}^{1}$. Also since $\varnothing \in A, B_{\sigma} \subseteq B^{\prime}$ and since $A$ is compatible with $I, B^{\prime} \subseteq I$. By Theorem 1.7 (i), there is a $\Pi_{2}^{0}$ set $C$ with $B \subseteq C \subseteq I$. Applying this to the components $\left\{B_{\xi}\right\}_{\xi<\omega_{1}}$ of $I$, we easily get an increasing family of $\Pi_{2}^{0}$ sets $\left\{C_{\xi}\right\}_{\xi<\omega_{1}}$ with union $I$, so $I$ is $\Pi_{2}^{0}$.

Related to Theorems 9 and 10 are two natural questions.

Q1. Is every calibrated thin $\Pi_{1}^{1} \sigma$-ideal of compact sets necessarily $\Pi_{2}^{0}$ ?

Q2. Is every calibrated thin $\Pi_{2}^{0} \sigma$-ideal of compact sets necessarily controlled?

Question 2 is some kind of weak version of the Maharam conjecture for control by measures (which is itself open). In the context of measures, a partial answer is given by the (second half) of the result of Dellacherie we quoted in 3.1. The same proof gives a similar partial result in the abstract frame.

Say that a $\sigma$-ideal of Borel sets $I$ on $E$ is regular if $I=I \cap K(E)$ is controlled, and normal if it is the intersection of regular $\sigma$-ideals. (This is the abstract version of $\gamma$ being a supremum of measures.)

THEOREM 13. Let I be a normal $\sigma$-ideal of Borel sets on a compact metrizable space $E$, with the inner approximation property. If $I \cap \mathcal{K}(E)=I$ is thin, then $I$ is controlled (hence I is regular).

Proof. The hypotheses imply that Borel $(E) / I$ has the c.c.c. As the countable intersection of regular $\sigma$-ideals is regular (by taking as control set the intersection of control sets), the result follows from the next lemma. 
LEMMA 14 (MOKовODZKI [DFM]). Let $I=\bigcap_{x \in X} I_{x}$ be a $\sigma$-ideal of Borel sets, and suppose Borel $(E) / I$ has the c.c.c. Then for some countable $Y \subseteq X, I=$ $\bigcap_{x \in Y} I_{x}$.

ProOF. Since Borel $(E) / I$ is a complete Boolean algebra, families of Borel sets admit ess. suprema modulo $I$. For each $x \in X$, let $B_{x}$ be an ess. supremum mod $I$ of $I_{x}$. Then $B_{x} \in I_{x}$, and if $A$ is Borel and $A-B_{x} \in I_{x}, A-B_{x} \in I$. Let now $B=\bigcup_{n} B_{x_{n}}^{c}$ be an ess. supremum $\bmod I$ of $\left\{B_{x}^{c}: x \in X\right\}$, and let $Y=\left\{x_{n}: n \in \omega\right\}$. We claim that $I=\bigcap_{x \in Y} I_{x}$. If $A \in \bigcap_{x \in Y} I_{x}$, then for each $n, A \cap B_{x_{n}}^{c} \in I_{x_{n}}$, hence $A \cap B_{x_{n}}^{c} \in I$. So it is enough to show $B^{c} \in I$. If not, there is $x \in X$ with $B^{c} \notin I_{x}$, hence $B^{c}-B_{x}=B_{x}^{c}-B \notin I_{x}$, and a fortiori $B_{x}^{c}-B \notin I$, contradicting the choice of $B$.

\section{REFERENCES}

[B] N. Bary, A treatise on trigonometric series, vols. 1 and 2, Macmillan, 1964.

$[\mathbf{B} \rightarrow$ J. P. Burgess and R. D. Mauldin, Conditional distributions and orthogonal measures, Ann. Proba. 9 (1981), 902-906.

[CM] D. Cenzer and R. D. Mauldin, Faithful extensions of analytic sets to Borel sets, Houston J. Math. 6 (1980), 19-29.

[Ch] G. Choquet, Theory of capacities, Ann. Inst. Fourier (Grenoble) 5 (1955), 131-295.

[Chr] J. P. R. Christensen, Necessary and sufficient conditions for the measurability of certain sets of closed subsets, Math. Ann. 200 (1973), 189-193.

[D1] C. Dellacherie, Ensembles analytiques, capacités, mesures de Hausdorff, Lecture Notes in Math., vol. 295, Springer-Verlag, Berlin and New York, 1972.

[D2] __, Capacités et processus stochastiques, Ergeb. Math. 67, Springer-Verlag, 1972.

[DFM] C. Dellacherie, D. Feyel and G. Mokobodzki, Intégrales de capacités fortement sous-additives, Sém. Prob. Strasbourg XVI, Lecture Notes in Math., vol. 920, Springer-Verlag, 1982, pp. 828.

[D3] C. Dellacherie, Appendice á l'exposé précédent, ibid., pp. 29-40.

[DM] C. Dellacherie and P. A. Meyer, Probabilités et potentiel, vol. 3, Hermann, Paris, 1984.

[Hi] G. Hillard, Une généralisation du théorème de Saint-Raymond sur les boréliens à coupes $K_{\sigma}$, C.R. Acad. Sci. Paris 288 (1970), 749-751.

[Hu] W. Hurewicz, Relativ Perfecte Teile von Punktmengen und Mengen (A), Fund. Math. (12), 1928.

[KS] J. P. Kahane and R. Salem, Ensembles parfaits et séries trigonométriques, Hermann, Paris, 1963.

[K1] R. Kaufman, Fourier transforms and descrpitive set theory, Mathematika 31 (1984), 336-339.

[K2] _ private communication, January 1985.

[KLSS] A. S. Kechris, A. Louveau, J. Saint-Raymond and J. Stern, Inaccessible cardinals and characterizations of Polish spaces (in preparation).

[KS] J. P. Kahane and R. Salem, Ensembles parfaits et séries trigonométriques, Hermann, Paris, 1963.

[Ku] K. Kuratowski, Topology, vols. 1 and 2, Academic Press, New York, 1966.

[L1] A. Louveau, Ensembles analytiques et boréliens dans les espaces produits, Astérisque 78 (1980).

[L2] _ Recursivity and capacity theory, Proc. Sympos. Pure Math., vol. 42, Amer. Math. Soc., Providence, R.I., 1985, pp. 285-301.

[MK] D. A. Martin and A. S. Kechris, Infinite games and effective descriptive set theory, Analytic Sets (C. A. Rogers et al., Eds.), Academic Press, 1980, pp. 404-469.

[StR1] J. Saint Raymond, Caractérisations d'espaces Polonais, d'après des travaux recents de J.P.R. Christensen et D. Preiss, Sém. Choquet $11^{\mathrm{e}}-12^{\mathrm{e}}$ annés, 1971-1973, no.5.

[StR2] _ L La structure borélienne d'Effros est-elle standard?, Fund. Math. 100 (1978), 201-210.

[S] R. M. Solovay, private communication, December 1983.

Department of Mathematics, California institute of Technology, PasaDENA, CALIFORNIA 91125

Equipe D'Analyse, Université Paris Vi, Tour 46-4Eme Ètage, 4, Place Jussieu, 75230, PARIS CEDEX 05 FRANCE 